\title{
Euglenophyta no Parque Estadual Delta do Jacuí, Rio Grande do Sul, Sul do Brasil. 3. Gênero Strombomonas Defl.
}

\author{
Sandra Maria Alves-da-Silva ${ }^{1}$ e Fernanda Cardoso Bridi ${ }^{2}$
}

Recebido em 10/03/2003. Aceito em 14/01/2004

\begin{abstract}
RESUMO - (Euglenophyta no Parque Estadual Delta do Jacuí, Rio Grande do Sul, Sul do Brasil. 3. Gênero Strombomonas Defl.). Foram identificados 34 táxons específicos e infra-específicos do gênero Strombomonas Defl. resultado do estudo de amostras qualitativas no Parque Estadual Delta do Jacuí, localizado a $29^{\circ} 56^{\prime}-30^{\circ} 03^{\prime} \mathrm{S}$ e $51^{\circ} 12^{\prime}-51^{\circ} 18^{\prime} \mathrm{W}$. As amostragens foram realizadas em 25 ambientes aquáticos em abril/1993 e janeiro/1994 e maio/1998 a setembro/1999. Strombomonas borystheniensis, S. ensifera, S. gibberosa, S. girardiana, S. scabra, S. triquetra var. torta e S. verrucosa foram os táxons mais freqüentes na área, ocorrendo em mais de $50 \%$ dos locais amostrados, destacando-se $S$. verrucosa pela ampla distribuição na área do Parque, com a ocorrência em $96 \%$ dos locais estudados. Strombomonas chodatti, S. cuneata, S. moreniensis, S. napiformis var. brevicollis são primeiras citações de ocorrência para o Estado e Brasil. S. confortii, S. fluviatilis var. major, S. morenensis, S. scabra var. intermedia e S. scabra var. ovata f. minor ocorrem exclusivamente na América do Sul. São realizadas considerações taxonômicas e ecológicas sobre o gênero, assim como é fornecida sua distribuição nacional e mundial.
\end{abstract}

Palavras-chave: taxonomia, Euglenophyta, Strombomonas, Sul do Brasil

ABSTRACT - (Euglenophyta in the Jacuí Delta State Park, Rio Grande do Sul State, Southern Brazil. 3. The genus Strombomonas Defl.). Thirty four specific and infra-specific taxa of the genus Strombomonas Defl. were identified as the result of the study of qualitative samples from Jacuí Delta State Park, that is located for $29^{\circ} 56^{\prime}-30^{\circ} 03^{\prime} \mathrm{S}$ and $51^{\circ} 12^{\prime}-51^{\circ} 18^{\prime} \mathrm{W}$. Sampling were carried out in 25 stations from April/1993 to January/1994 and May/1998 to September/1999. Strombomonas borystheniensis, S. ensifera, S. gibberosa, S. girardiana, S. scabra, S. triquetra var. torta and S. verrucosa were the most frequent taxa in the area, occuring in more than $50 \%$ of the samples, being distinguished $S$. verrucosa for its wide distribution in the area of the Park, with the occurrence in $96 \%$ of the studied places. Strombomonas chodatti, S. cuneata, S. moreniensis and S. napiformis var. brevicollis, were registered for the first time to the State and Brazilian territory. S. confortii, S. fluviatilis var. major, S. morenensis, S. scabra var. intermedia and S. scabra var. ovata f. minor are taxa that occur exclusively in South America. Taxonomic and ecological considerations are supplied as well as its national and world distribution.

Key words: taxonomy, Euglenophyta, Strombomonas, Southern Brazil

\section{Introdução}

As Euglenophyceae são algas com ampla distribuição ao redor do mundo, especialmente em ambientes continentais, e segundo Round (1983) podem ocorrer principalmente em águas com elevados teores de matéria orgânica, nitrogênio e fósforo.

Dentro dessa classe é encontrado o gênero Strombomonas, que foi proposto por Deflandre (1930) a partir da Subsecção Saccatae, série Asperae, senso Deflandre (1926), do gênero Trachelomonas. Desde a proposição do gênero Strombomonas muita polêmica tem sido levantada na separação destes dois gêneros. Tell \& Conforti (1988), ao trabalharem em microscópio eletrônico de varredura, observaram que somente o gênero Strombomonas aglutinava material em suas lóricas. Os autores propuseram, então, que espécies de Trachelomonas que aglutinassem partículas do meio em suas lóricas deveriam ser transferidas para Strombomonas. Apesar da controvérsia existente entre os dois gêneros, no presente trabalho adotou-se o sistema de Deflandre (1930) que se baseia fundamentalmente, em características morfológicas da lórica como as dimensões, forma e ornamentação da parede, utilizando-se também a relação entre o comprimento e a largura da lórica, forma e dimensões do cloroplasto, ausência ou presença e tipo de pirenóides.

O estudo foi realizado no Parque Estadual Delta do Jacuí, a $29^{\circ} 56^{\prime}-30^{\circ} 03^{\prime} \mathrm{S}$ e $51^{\circ} 12^{\prime}-51^{\circ} 25^{\prime} \mathrm{W}$ que faz parte da bacia do Guaíba, a qual constitui uma das

\footnotetext{
1 Museu de Ciências Naturais da Fundação Zoobotânica do Rio Grande do Sul, C. Postal 1188, CEP 90610-000, Porto Alegre, RS, Brasil

2 Bolsista de Iniciação Científica da Fundação de Amparo à Pesquisa do Rio Grande do Sul (FAPERGS), no Museu de Ciências Naturais. (Proc. 00504695)

3 Autores para correspondência: algas@ fzb.rs.org.br; gcferraz@cpovo.net
} 
mais expressivas áreas naturais da região metropolitana de Porto Alegre, capital do Estado do Rio Grande do Sul, abrangendo área de 1.7245 ha. O estudo da ficoflora do Parque fez parte do Programa para o Desenvolvimento Racional, Recuperação e Gerenciamento Ambiental da Bacia Hidrográfica do Guaíba (Pró-Guaíba). Com o objetivo de selecionar os locais mais representativos da área do Parque foram realizadas amostragens do fitoplâncton em 1993/1994 considerada como etapa piloto, resultando em dois trabalhos (Alves-da-Silva \& Ávila 1997; Alves-da-Silva \& Crossetti 1999), com a citação de sete espécies do gênero Strombomonas. As amostragens dentro do projeto Pró-Guaíba foram iniciadas somente em 1998. O presente trabalho é o resultado da análise de amostras de rede coletadas em 1993/1994 e 1998/1999, constituindo-se no quinto de uma série de publicações sobre a ficoflora de Euglenophyta do Parque.

Este estudo vem contribuir na ampliação do conhecimento taxonômico e ecológico, assim como a distribuição geográfica do gênero Strombomonas.

\section{Material e métodos}

Foram analisadas 85 amostras coletadas no período de abril/1993 e janeiro/1994 e de maio/1998 a setembro/1999, distribuídas em oito margens de ilhas, oito sacos, quatro desembocadura de rios (Caí, Gravataí, Jacuí e Sinos) e três canais, sendo encontrados especimes do gênero em somente 71 amostras.

Maiores detalhes da área de estudo e da metodologia utilizada encontram-se em Alves-da-Silva \& Hahn (2004).

Para a identificação específica e infra-específica dos táxons foram utilizadas obras como Deflandre (1930), Conrad \& Van Mell (1952), Huber-Pestalozzi (1955), Starmach (1983), Tell \& Conforti (1986), além de trabalhos recentes do grupo.

São fornecidas informações da variação morfométrica dos táxons, descrições apenas das feições morfológicas observadas, ilustrações somente dos táxons ainda não citados para área, relação entre o comprimento e a largura da lórica $=\mathrm{Rc} / \mathrm{l}$, comentários e dados de algumas variáveis abióticas em que cada táxon ocorreu na área de estudo (Tab. 1).

As amostras que apresentam o gênero Strombomonas estão depositadas no acervo do Herbário Prof. Dr. Alarich R.H. Schultz (HAS), no Museu de Ciências Naturais da Fundação Zoobotânica do Rio Grande do Sul (Tab. 2).

Tabela 1. Variáveis abióticas e ambientais onde ocorreram os táxons de Strombomonas, no Parque Estadual Delta do Jacuí, RS, em 1993/ 1994 e 1998/1999. NM = não medido.

\begin{tabular}{|c|c|c|c|c|c|c|}
\hline \multirow[b]{2}{*}{ Táxons } & \multicolumn{6}{|c|}{ Variáveis abióticas } \\
\hline & $\mathrm{pH}$ & $\begin{array}{c}\text { Temperatura } \\
\left({ }^{\circ} \mathrm{C}\right)\end{array}$ & $\begin{array}{l}\text { Amônia } \\
\left(\mu \mathrm{g} \cdot \mathrm{L}^{-1}\right)\end{array}$ & $\begin{array}{l}\text { Nitrito } \\
\left(\mu \mathrm{g} . \mathrm{L}^{-1}\right)\end{array}$ & $\begin{array}{l}\text { Nitrato } \\
\left(\mu \mathrm{g} . \mathrm{L}^{-1}\right)\end{array}$ & $\begin{array}{c}\text { Transparência } \\
(\mathrm{cm})\end{array}$ \\
\hline S. acuminata var. amphora & 6,2 & $19-22$ & NM & 20 & 900 & $\mathrm{NM}$ \\
\hline S. borystheniensis & $5-8,2$ & $13,1-30,3$ & $\mathrm{NM}$ & $1-131$ & $500-2100$ & $10-80$ \\
\hline S. chodatii & 6,1 & 22,1 & 287 & 10 & 700 & 30 \\
\hline S. confortii & 6,8 & $23,1-23,2$ & $60-360$ & $107-131$ & $1300-2100$ & $25-30$ \\
\hline S.costata & $6,3-6,8$ & $22-30,3$ & 1800 & $7-10$ & 1100 & $10-80$ \\
\hline S. cuneata & 6,8 & 23,2 & 360 & 131 & 1300 & 30 \\
\hline S. deflandrei & $6,8-7$ & $13-30$ & 390 & $19-25$ & 1200 & $15-30$ \\
\hline S. elegans & $6,5-8,8$ & $15,2-30,3$ & $15-140$ & $7-48$ & $1100-1400$ & $20-40$ \\
\hline S. ensifera & $6,5-7,6$ & $23,1-30$ & $60-1200$ & $23-131$ & $800-2100$ & $25-80$ \\
\hline S. fluviatilis var. fluviatilis & $5-7,6$ & $17-30,3$ & $15-1200$ & $1-107$ & $600-2100$ & $10-80$ \\
\hline S. fluviatilis var. laevis & $\mathrm{NM}$ & 28 & $\mathrm{NM}$ & $\mathrm{NM}$ & NM & NM \\
\hline S. fluviatilis var. major & $6,8-7,4$ & 27 & 1200 & 60 & 800 & $30-80$ \\
\hline S.globulosa & $5,6-7,4$ & $27-28$ & $\mathrm{NM}$ & $\mathrm{NM}$ & $\mathrm{NM}$ & $30-40$ \\
\hline S. gibberosa & $6-8,8$ & $15,2-30,3$ & $10-1800$ & $1-131$ & $70-2100$ & $10-80$ \\
\hline S. girardiana & $5,5-8,8$ & $13,1-30,3$ & $60-1300$ & $5-131$ & $700-2100$ & $20-70$ \\
\hline S. lanceolata & $5,6-7,6$ & $22-30,3$ & 166 & $1-25$ & 300 & $25-50$ \\
\hline S. maxima & $5,6-7,6$ & $22-30,3$ & $60-1200$ & $7-131$ & $700-2100$ & $25-80$ \\
\hline S. moreniensis & 6,1 & 22,1 & 287 & 10 & 700 & $\begin{array}{l}30 \\
\text { continua }\end{array}$ \\
\hline
\end{tabular}


Tabela 1 (continuação)

\begin{tabular}{|c|c|c|c|c|c|c|}
\hline \multirow[b]{2}{*}{ Táxons } & \multicolumn{6}{|c|}{ Variáveis abióticas } \\
\hline & $\mathrm{pH}$ & $\begin{array}{c}\text { Temperatura } \\
\left({ }^{\circ} \mathrm{C}\right)\end{array}$ & $\begin{array}{l}\text { Amônia } \\
\left(\mu \mathrm{g} . \mathrm{L}^{-1}\right)\end{array}$ & $\begin{array}{l}\text { Nitrito } \\
\left(\mu \mathrm{g} \cdot \mathrm{L}^{-1}\right)\end{array}$ & $\begin{array}{l}\text { Nitrato } \\
\left(\mu \mathrm{g} \cdot \mathrm{L}^{-1}\right)\end{array}$ & $\begin{array}{c}\text { Transparência } \\
(\mathrm{cm})\end{array}$ \\
\hline S. napiformis var. brevicollis & $6,1-6,2$ & $18-26$ & $90-120$ & $2-4$ & $400-800$ & $10-35$ \\
\hline S. rotunda & $5-7$ & $23,2-30,3$ & 360 & $7-131$ & 1300 & $30-50$ \\
\hline S. scabra var. coronata & 7 & 30 & 360 & & 9 & 25 \\
\hline S. scabra var. intermedia & $6-7,6$ & $23-30,3$ & $360-1800$ & $10-131$ & $600-1300$ & $10-30$ \\
\hline S.scabra var. labiata & $6,7-7$ & $28,4-30,3$ & $\mathrm{NM}$ & $9-10$ & $\mathrm{NM}$ & $25-30$ \\
\hline S. scabra var. longicollis & 6 & $23-30$ & 1300 & 600 & 38 & 25 \\
\hline S. scabra var. ovata f. minor & $5,9-7$ & $13,1-30,3$ & $12-600$ & $1-50$ & $500-1500$ & $20-45$ \\
\hline S. scabra var. ovata & $5-8,8$ & $17,4-30,3$ & $360-1300$ & $7-131$ & $600-1300$ & $25-70$ \\
\hline S. scabra var. scabra & $5-7,6$ & $13,1-30$ & $10-1300$ & $1-131$ & $300-2100$ & $10-80$ \\
\hline S. schauinslandii & $7-7,4$ & $27-30$ & $\mathrm{NM}$ & $19-25$ & $\mathrm{NM}$ & 30 \\
\hline S. tetraptera var. gallica & $\mathrm{NM}$ & $28-29$ & $\mathrm{NM}$ & $\mathrm{NM}$ & $\mathrm{NM}$ & $\mathrm{NM}$ \\
\hline S. treubii & $5-7$ & $15,5-30$ & $12-1200$ & $1-60$ & $300-900$ & $10-80$ \\
\hline S. triqueta var. torta & $5-7,4$ & $17-30,3$ & $12-1200$ & 4-107 & $500-2100$ & $10-80$ \\
\hline S. urceolata & $5-6,4$ & $22,1-28,4$ & $287-1300$ & $1-38$ & $600-700$ & $25-50$ \\
\hline S. verrucosa var. verrucosa & $5-8,8$ & $12,4-30,3$ & $10-1800$ & $1-131$ & $300-2100$ & $10-80$ \\
\hline S. verrucosa var. genuina & $5,5-7,4$ & $20-30$ & 1300 & $9-38$ & $600-1100$ & $25-70$ \\
\hline
\end{tabular}

Táxons

\begin{tabular}{cccccc}
\hline $\begin{array}{c}\text { Ortofosfato } \\
\left(\mu \mathrm{g} . \mathrm{L}^{-1}\right)\end{array}$ & $\begin{array}{c}\text { Sílica } \\
\left(\mathrm{mg} \cdot \mathrm{L}^{-1}\right)\end{array}$ & $\begin{array}{c}\text { Matéria } \\
\text { orgânica } \\
\left(\mathrm{mg} . \mathrm{L}^{-1}\right)\end{array}$ & $\begin{array}{c}\text { Oxigênio } \\
\text { dissolvido } \\
\left(\mathrm{mg} . \mathrm{L}^{-1}\right)\end{array}$ & $\begin{array}{c}\text { Demanda } \\
\text { bioquímica de } \\
\text { oxigênio } \\
\left(\mathrm{mg} . \mathrm{L}^{-1}\right)\end{array}$ & $\begin{array}{c}\text { Coliformes } \\
\text { fecais }\end{array}$ \\
& & & & $\left.\mathrm{mg} . \mathrm{L}^{-1} / 100 \mathrm{~mL}\right)$
\end{tabular}

\begin{tabular}{|c|c|c|c|c|c|c|}
\hline S. acuminata var. amphora & 290 & 37 & 4,7 & 5,4 & 2,5 & 8000 \\
\hline S. borystheniensis & $30-11500$ & $8,1-27$ & $1,4-9,5$ & $1-9,2$ & $0,2-2,9$ & $10-1800$ \\
\hline S. chodatii & 120 & 16,4 & 3,3 & 8,2 & 0,6 & $\mathrm{NM}$ \\
\hline S. confortii & $340-530$ & $8,1-8,4$ & $3,6-3,7$ & $4,4-5,3$ & $1-2,4$ & $2800-20000$ \\
\hline S.costata & 11500 & 17 & $3,4-9,5$ & $1-6,5$ & $1,4-2,9$ & 170 \\
\hline S. cuneata & 530 & 8,1 & 3,7 & 4,4 & 2,4 & 20000 \\
\hline S. deflandrei & 240 & 7,5 & $3,3-6$ & $4,4-6,9$ & $0,6-1,6$ & $110-11000$ \\
\hline S. elegans & $220-240$ & $7,2-9,2$ & $1,6-6$ & $5,6-6,9$ & $0,3-1,4$ & $110-800$ \\
\hline S. ensifera & $90-1440$ & $8,1-27$ & $3,3-3,8$ & $1,1-5,6$ & $0,6-2,4$ & $80-20000$ \\
\hline S. fluviatilis var. fluviatilis & $110-1440$ & $7,2-16$ & $1,4-4,8$ & $1,1-8,5$ & $0,2-1,8$ & $110-16000$ \\
\hline S. fluviatilis var. laevis & NM & $\mathrm{NM}$ & $\mathrm{NM}$ & NM & $\mathrm{NM}$ & NM \\
\hline S. fluviatilis var. major & 1440 & 16 & 4,8 & 1,1 & 1,2 & NM \\
\hline S. globulosa & 1,3 & $\mathrm{NM}$ & 7,3 & 1,3 & NM & NM \\
\hline S. gibberosa & $60-11500$ & $15,2-30,3$ & $1,8-9,5$ & $1-9,2$ & $0,1-2,9$ & $90-20000$ \\
\hline S. girardiana & $30-1900$ & $8,1-16$ & $3,3-8$ & $1,1-9,2$ & $0,1-2,4$ & $20-20000$ \\
\hline S. lanceolata & 60 & 11 & $3,1-7,3$ & $1,3-6,9$ & $0,3-1,4$ & $110-800$ \\
\hline S. maxima & $120-1440$ & $8,1-16,4$ & $3,3-7,3$ & $1,1-9,2$ & $0,6-2,4$ & $110-20000$ \\
\hline S. moreniensis & 120 & 16,4 & 3,3 & 8,2 & 0,6 & $\mathrm{NM}$ \\
\hline S.napiformis var. brevicollis & $110-160$ & $13,3-16$ & $4-4,6$ & $7,6-8,3$ & $0,7-1,6$ & $\mathrm{NM}$ \\
\hline S. rotunda & 530 & 8,1 & $1,4-3,8$ & $4,4-6,9$ & $1,3-2,4$ & $110-20000$ \\
\hline S. scabra var. coronata & NM & $\mathrm{NM}$ & 3,1 & 6,1 & 1 & 140 \\
\hline S. scabra var. intermedia & $530-11500$ & $8,1-17$ & $3,7-9,5$ & $1-6,9$ & $1,1-2,9$ & $20-20000$ \\
\hline S. scabra var. labiata & $\mathrm{NM}$ & $\mathrm{NM}$ & $3,1-3,9$ & $6,1-6,9$ & $1-1,3$ & $20-140$ \\
\hline S. scabra var. longicollis & 1900 & 15 & 8 & 2,5 & 1,7 & NM \\
\hline S. scabra var. ovata f. minor & $90-460$ & $6,4-27$ & $1,6-4,6$ & $3,1-8,2$ & $0,1-2,1$ & $20-16000$ \\
\hline S. scabra var. ovata & $360-1900$ & $8,1-15$ & $1,4-8$ & $2,4-8$ & $0,6-1,7$ & $110-20000$ \\
\hline
\end{tabular}


Tabela 1 (continuação)

\begin{tabular}{|c|c|c|c|c|c|c|}
\hline \multirow[b]{2}{*}{ Táxons } & \multicolumn{6}{|c|}{ Variáveis ambientais } \\
\hline & $\begin{array}{l}\text { Ortofosfato } \\
\qquad\left(\mu \mathrm{g} . \mathrm{L}^{-1}\right)\end{array}$ & $\begin{array}{c}\text { Sílica } \\
\left(\mathrm{mg} . L^{-1}\right)\end{array}$ & $\begin{array}{l}\text { Matéria } \\
\text { orgânica } \\
\left(\mathrm{mg} \cdot \mathrm{L}^{-1}\right)\end{array}$ & $\begin{array}{l}\text { Oxigênio } \\
\text { dissolvido } \\
\left(\mathrm{mg} . \mathrm{L}^{-1}\right)\end{array}$ & $\begin{array}{c}\text { Demanda } \\
\text { bioquímica de } \\
\text { oxigênio } \\
\left(\mathrm{mg} . \mathrm{L}^{-1}\right)\end{array}$ & $\begin{array}{c}\text { Coliformes } \\
\text { fecais } \\
\left(\mathrm{mg} \cdot \mathrm{L}^{-1} / 100 \mathrm{~mL}\right)\end{array}$ \\
\hline S. scabra var. scabra & $60-1900$ & $8,1-20$ & $1,4-8$ & $1,1-9,2$ & $0,2-2,4$ & $40-20000$ \\
\hline S. schauinslandii & NM & NM & $3,3-3,8$ & $5,6-6,9$ & $0,6-1,3$ & $110-800$ \\
\hline S. tetraptera var. gallica & NM & $\mathrm{NM}$ & $\mathrm{NM}$ & $\mathrm{NM}$ & $\mathrm{NM}$ & $\mathrm{NM}$ \\
\hline S. treubii & $60-1520$ & $4,9-34$ & $1,4-7,9$ & $1,1-8,2$ & $0,2-6,4$ & $40-28000$ \\
\hline S. triqueta var. torta & $30-1900$ & $8,4-16$ & $1,4-8$ & $1,1-8,5$ & $0,2-2,2$ & $110-16000$ \\
\hline S. urceolata & $120-1900$ & $15-16,4$ & $1,4-8$ & $2,5-8,2$ & $0,6-1,7$ & NM \\
\hline S. verrucosa var. verrucosa & $30-11500$ & $4,7-17$ & $1,4-9,5$ & $1-9,2$ & $0,1-3,2$ & $20-28000$ \\
\hline S.verrucosa var. genuina & $530-1900$ & $5,5-7,4$ & $3,1-8$ & $2,4-7,9$ & $1,-2,4$ & $110-170$ \\
\hline
\end{tabular}

Tabela 2. Locais e data das coletas de amostras de água no Parque Estadual Delta de Jacuí, Rio Grande do Sul e o número de registro das amostras depositadas no Herbário Prof. Dr. R.H. Schultz (HAS).

\begin{tabular}{|c|c|c|c|c|c|}
\hline HAS & Estações de coleta & Data & HAS & Estações de coleta & Data \\
\hline 34632 & Saco do Cabral $=$ SC & $7 / 5 / 98$ & 26081 & Saco da Pólvora=SP & $13 / 4 / 93$ \\
\hline 34791 & Saco do Cabral $=\mathrm{SC}$ & $15 / 9 / 98$ & 34803 & Saco da Pólvora=SP & $15 / 9 / 98$ \\
\hline 34792 & Saco do Cabral $=$ SC & $15 / 9 / 98$ & 34817 & Saco da Pólvora=SP & $10 / 11 / 98$ \\
\hline 34805 & Saco do Cabral $=\mathrm{SC}$ & $10 / 11 / 98$ & 34893 & Saco da Pólvora=SP & $5 / 4 / 99$ \\
\hline 34881 & Saco do Cabral=SC & $5 / 4 / 99$ & 34955 & Saco da Pólvora=SP & $14 / 6 / 99$ \\
\hline 34945 & Saco do Cabral $=$ SC & $14 / 6 / 99$ & 34647 & Foz Rio Gravataí=FG & $26 / 2 / 98$ \\
\hline 34959 & Saco do Cabral $=$ SC & $27 / 9 / 99$ & 34665 & Foz Rio Gravataí=FG & $19 / 5 / 98$ \\
\hline 34793 & Saco das Garças $=\mathrm{SG}$ & $15 / 9 / 98$ & 34819 & Foz Rio Gravataí=FG & $16 / 11 / 98$ \\
\hline 34807 & Saco das Garças=SG & $10 / 11 / 98$ & 34651 & Foz Rio dos Sinos=FS & $26 / 2 / 98$ \\
\hline 34883 & Saco das Garças $=\mathrm{SG}$ & $5 / 4 / 99$ & 34667 & Foz Rio dos Sinos=FS & $19 / 5 / 98$ \\
\hline 34947 & Saco das Garças $=$ SG & $14 / 6 / 99$ & 34712 & Foz Rio dos Sinos=FS & $11 / 8 / 98$ \\
\hline 34961 & Saco das Garças $=\mathrm{SG}$ & 27/9/99 & 34822 & Foz Rio dos Sinos=FS & $16 / 11 / 98$ \\
\hline 34635 & Ilha do Serafim=IS & 7/5/99 & 34654 & Foz Rio Caí=FC & $26 / 2 / 98$ \\
\hline 34795 & Ilha do Serafim=IS & $15 / 9 / 98$ & 34715 & Foz Rio Caí=FC & $11 / 8 / 98$ \\
\hline 34809 & Ilha do Serafim=IS & $10 / 11 / 98$ & 34825 & Foz Rio Caí=FC & $16 / 11 / 98$ \\
\hline 34885 & Ilha do Serafim=IS & $5 / 4 / 99$ & 34657 & Foz Rio Jacuí=FJ & $26 / 2 / 98$ \\
\hline 34963 & Ilha do Serafim=IS & 27/9/99 & 34720 & Foz Rio Jacuí=FJ & $11 / 8 / 98$ \\
\hline 34637 & Canal Feliz=CF & $7 / 5 / 99$ & 34831 & Foz Rio Jacuí=FJ & $16 / 11 / 98$ \\
\hline 34811 & Canal Feliz $=\mathrm{CF}$ & $10 / 11 / 98$ & 34992 & Saco dos Cachorros $=\mathrm{SCA}$ & $27 / 12 / 93$ \\
\hline 34887 & Canal Feliz $=\mathrm{CF}$ & $5 / 4 / 99$ & 26327 & Ilha do Cravo=ICR & $15 / 12 / 93$ \\
\hline 34965 & Canal Feliz $=\mathrm{CF}$ & 27/9/99 & 34998 & Ilha do Cravo=ICR & 27/12/99 \\
\hline 26087 & Saco do Quilombo=SQ & $13 / 4 / 93$ & 34990 & Ilha da Formiga=IFO & 27/12/99 \\
\hline 26339 & Saco do Quilombo=SQ & $15 / 12 / 93$ & 26333 & Ilha da Siqueira=ISQ & $15 / 12 / 93$ \\
\hline 34799 & Saco do Quilombo=SQ & $15 / 9 / 98$ & 34994 & Ilha da Siqueira=ISQ & $27 / 12 / 99$ \\
\hline 34813 & Saco do Quilombo=SQ & $10 / 11 / 98$ & 26329 & Ilha da Cabeçuda=IC & $15 / 12 / 93$ \\
\hline 34889 & Saco do Quilombo=SQ & $5 / 4 / 99$ & 34996 & Ilha da Cabeçuda=IC & $27 / 12 / 99$ \\
\hline 34953 & Saco do Quilombo=SQ & $14 / 6 / 99$ & 26342 & Canal das Balseiras $=\mathrm{CB}$ & $15 / 12 / 93$ \\
\hline 26079 & Saco do Jacaré=SJ & $13 / 4 / 93$ & 34908 & Canal das Balseiras $=\mathrm{CB}$ & 19/1/99 \\
\hline 34643 & Saco do Jacaré=SJ & $7 / 5 / 99$ & 26347 & Foz Arroio Guaíba=FAG & $4 / 1 / 94$ \\
\hline 34801 & Saco do Jacaré $=$ SJ & $15 / 9 / 98$ & 34957 & Saco do Jacaré $=$ SJ & $14 / 6 / 99$ \\
\hline 34815 & Saco do Jacaré $=$ SJ & $10 / 11 / 98$ & 34922 & Saco Santa Cruz=SSC & $19 / 1 / 99$ \\
\hline 34891 & Saco do Jacaré $=$ SJ & $5 / 4 / 99$ & 34910 & Ilha das Pombas=IP & $19 / 1 / 99$ \\
\hline 34969 & Saco do Jacaré $=$ SJ & 27/9/99 & 34914 & Ilha Grande Marinheiros=IM & $19 / 1 / 99$ \\
\hline \multirow[t]{2}{*}{26070} & Foz Rio Gravataí=FG & $12 / 04 / 93$ & 34916 & Saco do Ferraz & $19 / 1 / 99$ \\
\hline & & & 34920 & Ilha das Flores & $19 / 1 / 99$ \\
\hline
\end{tabular}




\section{Resultados}

Foram encontrados representantes do gênero Strombomonas em 71 amostras analisadas, resultando na identificação de 34 táxons específicos e infraespecíficos apresentados a seguir.

Euglenophyta

Euglenophyceae

Euglenales

Gênero Strombomonas Defl.

1. Strombomonas acuminata (Schm.) Defl. var. amphora Playf., Proc. Linn. Soc. N.S.W. 40(1): 41, pl. 5, fig. 3.1915.

Fig. 1-2.

Lórica elíptica a ovada, 46,2-47 $\mu \mathrm{m}$ compr., ca. $24 \mu \mathrm{m}$ larg., $\mathrm{Rc} / \mathrm{l}=1,9$; vista polar amplamente elíptica; pólo anterior gradativamente atenuado em colarinho cilíndrico, alargado na extremidade, bordo crenulado, ca. de 3,7 $\mu \mathrm{m}$ alt. e 7,4-8,3 $\mu \mathrm{m}$ larg.; pólo posterior abruptamente atenuado em processo caudal hialino, cônico, reto ou levemente curvado, ca. de $9 \mu \mathrm{m}$ compr.; parede verrucosa, castanho amarelada; cloropastos numerosos, discóides, 2,8-4,0 $\mu \mathrm{m}$ diâm.; flagelo ca. uma vez o comprimento da lórica.

Material examinado: HAS 26079, HAS 34961.

Distribuição no Brasil: Amazonas (Conforti 1993). Rio Grande do Sul (Alves-da-Silva 1998).

Distribuição geográfica: América do Sul, Ásia, Oceania.

2. Strombomonas borystheniensis (Roll) Pop., Flora plant. Cryptog. 3(1):206, pl. 20, fig 15. 1966.

Fig. 3-5.

Lórica oblonga ou amplamente elíptica, 24-34,2 $\mu \mathrm{m}$ compr., 21,3-26 $\mu \mathrm{m}$ larg., $\mathrm{Rc} / \mathrm{l}=1,2-1,4$; pólo anterior terminando em colarinho baixo, oblíquo, bordo

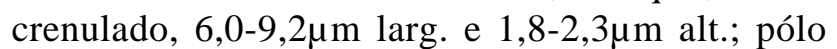
posterior arredondado; parede espessa, granulosa ou rugosa, coloração castanho-claro a médio; cloropastos discóides ou bastoniformes até quase $5 \mu \mathrm{m}$ diâm.

Material examinado: HAS 26079, HAS 26327, HAS 26333, HAS 26347, HAS 263448, HAS 34635, HAS 34647, HAS 34651, HAS 34654, HAS 34657, HAS 34791, HAS 34792, HAS 34801, HAS 34805 , HAS 34807, HAS 34809, HAS 34811, HAS 34815 , HAS 34817, HAS 34819, HAS 34822, HAS 34831, HAS 34881, HAS 34883, HAS 34885, HAS 34887 , HAS 34889, HAS 34893, HAS 34908, HAS 34910,
HAS 34924, HAS 34947, HAS 34992, HAS 34994 , HAS 34996, HAS 34998.

Distribuição no Brasil: Amazonas (Conforti 1993). Rio Grande do Sul (Alves-da-Silva \& Torres 1994; Alves-da-Silva 1998).

Distribuição geográfica: América do Sul, Ásia, Europa.

3. Strombomonas chodatii (Skv.) Defl., Arch. Protistenk. 69(3): 588, fig. 74-75. 1930.

Fig. 6.

Lórica oblonga ou trapezoidal, irregular, levemente mais larga no pólo anterior; 58,3-65 $\mu \mathrm{m}$ compr., ca. $25 \mu \mathrm{m}$ larg., Rc/l=2,3-2,6; pólo anterior atenua-se em colarinho baixo, ca. $4,6 \mu \mathrm{m}$ alt. e ca. 7,4 $\mu \mathrm{m}$ larg.; pólo posterior atenua-se abruptamente em processo caudal levemente curvo, inclinado, de 9,2-11 $\mu \mathrm{m}$ compr.; parede rugosa, hialina.

Material examinado: HAS 34811.

Distribuição no Brasil: Mato Grosso (DeLamonica-Freire et al. 1992).

Distribuição geográfica: América do Sul, Ásia, Europa, Oceania.

Comentários: espécie rara na área, sendo observados apenas três indivíduos, todos sem estruturas internas, sendo observada somente a lórica. Pode ser uma espécie alóctone à área. Segundo Tell e Conforti (1986) é uma espécie encontrada em águas quentes, no presente estudo foi encontrada em temperatura de $22^{\circ} \mathrm{C}$.

Primeira citação de ocorrência da espécie para o Estado e segunda para o país.

4. Strombomonas confortii Zaloc., Cryptogamie Algol. 12(4): 273. 1991.

Fig. 7-11.

Lórica cônica 39-51 $\mu \mathrm{m}$ compr., 23-33,3 $\mu \mathrm{m}$ larg.; $\mathrm{Rc} / 1=1,1-1,5$; vista polar circular; pólo anterior mais estreito que o pólo posterior, com uma expansão aliforme transversal que rodeia toda lórica na sua base; colarinho reto ou ligeiramente inclinado, 5,5-6,4 $\mu \mathrm{m}$ compr. e ca. 7,5 $\mu \mathrm{m}$ larg.; processo caudal levemente oblíquo $15-17 \mu \mathrm{m}$ compr.; parede espessa, irregular, castanho-clara a castanho-avermelhada; cloroplastos numerosos, bastoniformes, ca. $2 \mu \mathrm{m}$ compr.; pirenóides internos; flagelo uma vez o comprimento da lórica.

Material examinado: HAS 34881, HAS 34885.

Distribuição no Brasil: nova citação para Estado e país.

Distribuição geográfica: espécie encontrada exclusivamente na América do Sul 


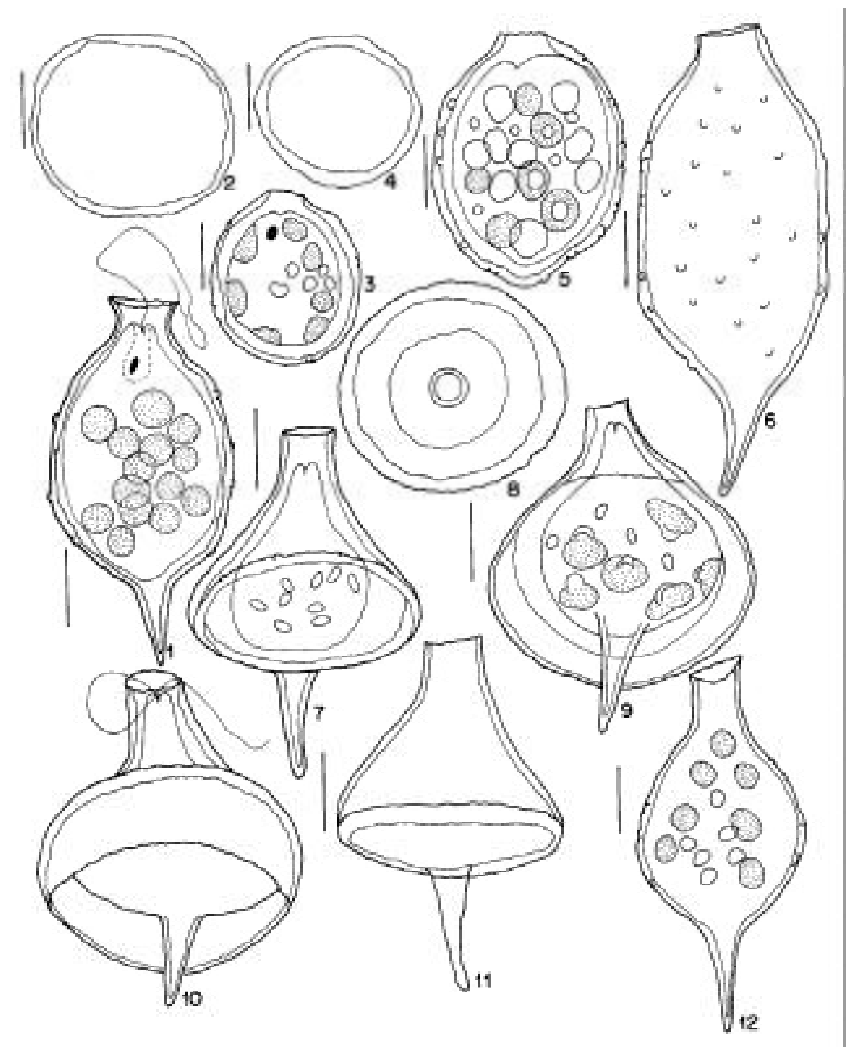

Figuras 1-12. 1-2. Strombomonas acuminata (Schm.) Defl. var. amphora Playf. 2. Vista polar. 3-5. Strombomonas borystheniensis (Roll) Pop. 4. Vista polar. 6. Strombomonas chodatii (Skv.) Defl. 7-11. Strombomonas confortii Zaloc. 8. Vista polar. 12. Strombomonas costata Defl. Escala $=10 \mu \mathrm{m}$.

Comentários: alguns exemplares apresentaram-se ligeiramente menores que o citado por Zalocar (1991: 47-48 $\mu \mathrm{m} \times 38-38,5 \mu \mathrm{m})$, porém a expansão aliforme transversal na lórica a diferencia de qualquer outra espécie de Strombomonas.

Foram encontrados alguns indivíduos com pirenóides internos, não descritos para esta espécie, informação que amplia o conhecimento deste táxon.

5. Strombomonas costata Defl., Arch. Protistenk. 69(3): 589, fig. 76-80. 1930.

Fig. 12.

Lórica elíptica a ovada, às vezes rombóides; 54-62 $\mu \mathrm{m}$ compr., 23-27 $\mu \mathrm{m}$ larg., Rc/l = 2,2-2,3; pólo anterior gradativamente atenuado em colarinho cilíndrico, alargado na extremidade, bordo crenulado, $4,0-6,5 \mu \mathrm{m}$ alt. e 7,4-9,2 $\mu \mathrm{m}$ larg.; pólo posterior abruptamente atenuado em processo caudal hialino, cônico, reto e levemente curvado $12-14 \mu \mathrm{m}$ compr.; parede levemente rugosa, hialina a castanho-claro amarelada; cloroplastos numerosos, grandes, ca. $5 \mu \mathrm{m}$.
Material examinado: HAS 26079, HAS 26327, HAS 34819, HAS 34916, HAS 34998.

Distribuição no Brasil: Amazonas (Conforti 1993). Rio de Janeiro (Souza 2002)

Distribuição geográfica: América do Sul, Europa.

Comentários: apesar de Deflandre (1930) mencionar que a espécie possui vista apical lobulada, Conforti (comunicação pessoal), comentou que muitos dos indivíduos encontrados na Argentina não apresentam reentrâncias lobuladas em vista apical, tal qual, o material encontrado no Parque.

Primeira citação da espécie para o Estado e terceira para o país.

6. Strombomonas cuneata (Playf.) Defl., Arch. Protistenk. 69(3): 594, fig. 89. 1930.

Fig. 13.

Lórica trapezoidal, ca. de $51 \mu \mathrm{m}$ compr., ca. $23,1 \mu \mathrm{m}$ larg., $\mathrm{Rc} / \mathrm{l}=2,2$; região central mais alargada; pólo anterior subtriangular estreitando-se rapidamente em colarinho cilíndrico, levemente reto, bordo levemente crenulado, ca. $12 \mu \mathrm{m}$ compr. e $7 \mu \mathrm{m}$ larg.; pólo posterior atenua-se rapidamente em processo caudal reto ca. $12 \mu \mathrm{m}$ compr.; parede levemente escabrosa, castanho-clara.

Material examinado: HAS 34881.

Distribuição no Brasil: nova citação para Estado e país.

Distribuição geográfica: Oceania.

Comentários: espécie considerada rara, tendo sido encontrada somente no Saco do Cabral, no outono, com um indivíduo por lâmina.

7. Strombomonas deflandrei (Roll) Defl., Arch. Protistenk. 69(3): 575, fig. 37. 1930.

Fig. 14.

Lórica elíptica a ovada, ca. 33,3 $\mu$ m compr., ca. $20,3 \mu \mathrm{m}$ larg., $\mathrm{Rc} / \mathrm{l}=1,6$; vista polar arredondada; pólo anterior alargado, colarinho curto, às vezes alargado na extremidade distal, reto ou oblíquo, ca. 2,7 $\mu \mathrm{m}$ compr. e 5,5 $\mu \mathrm{m}$ larg.; pólo posterior acuminado abruptamente em processo caudal cônico, reto ca. $4 \mu \mathrm{m}$ compr.; parede levemente rugosa; cloroplastos numerosos, discóides, ca. $3 \mu \mathrm{m}$ diâm.

Material examinado: HAS 26333, HAS 34945, HAS 34992, HAS 34994.

Distribuição no Brasil: Amazonas (Conforti 1993). Rio Grande do Sul (Alves-da-Silva \& Ferraz 1991; Franceschini 1992).

Distribuição geográfica: América do Norte, América do Sul, Ásia, Europa. 
8. Strombomonas elegans Conf. \& Joo, Cryptogamie Algol. 15 (4): 275, fig. 74a-d. 1994.

Lórica elíptica 28-33,3 $\mu$ m compr., 10-11,5 $\mu$ m larg., $\mathrm{Rc} / 1=2,4-3,3$; pólo anterior terminado em colarinho cilíndrico, extremidade distal divergente com bordo irregular, 4,6-5,0 $\mu \mathrm{m}$; pólo posterior levemente atenuado em processo caudal cônico, 2,7-4,6 $\mu \mathrm{m}$; parede verrucosa, hialina a amarelada; cloroplastos discóides ca. $2,7 \mu \mathrm{m}$; pirenóides internos presentes.

Material examinado: HAS 26342, HAS 34891, HAS 34908, HAS 34910, HAS 34957, HAS 34994, HAS 34998.

Distribuição no Brasil: Amazonas (Conforti 1993). Rio Grande do Sul (Alves-da-Silva \& Crosseti 1999).

Distribuição geográfica: América do Norte, América do Sul.

9. Strombomonas ensifera (Daday) Defl., Arch. Protistenk. 69(3): 601. 1930.

Fig. 15-16.

Lórica rombóide, 73-93,2 $\mu \mathrm{m}$ compr., 35-44 $\mu \mathrm{m}$ larg., $\mathrm{Rc} / \mathrm{l}=2,1$; parte equatorial alargada com ângulos ligeiramente arredondados; pólo anterior atenuado em colarinho cilindrico, truncado, oblíquo, ca. $7 \mu \mathrm{m}$; pólo posterior atenuado gradativamente em processo caudal longo ca. $30 \mu \mathrm{m}$; parede delgada, castanha-clara; cloroplastos numerosos, discóides, de 3,7-4,6 $\mu$ m diâm.; grãos de paramidos numerosos, arredondados ou alongados.

Material examinado: HAS 26327, HAS 26333, HAS 26342, HAS 34822, HAS 34881, HAS 34885, HAS 34908, HAS 34922, HAS 34992.

Distribuição no Brasil: Amazonas (Uherkovich \& Schmidt 1974; Conforti 1993; Bittencourt-Oliveira 1997). Mato Grosso (Menezes \& Fernandes 1987). Paraná (Jati \& Train 1994). Rio Grande do Sul (Alvesda-Silva 1988). Rio de Janeiro (Menezes 1994).

Distribuição geográfica: América do Norte, América do Sul, Ásia, Europa.

Comentários: Deflandre (1930) deu para esta espécie, dimensões de $120-134 \times 38-55 \mu \mathrm{m}$; os exemplares examinados apresentaram menores dimensões no comprimento do que as citadas por este autor, entretanto a morfologia geral da célula é semelhante à descrição da espécie.

Na Argentina Tell \& Conforti (1986) citaram que esta variedade só é conhecida em regiões quentes a temperadas em corpos d'água muito eutróficos.

10. Strombomonas fluviatilis (Lemm.) Defl. var. fluviatilis, Arch. Protistenk. 69(3): 580, fig. 52-53. 1930. Fig. 17-19.
Lórica elíptica, 27-39 $\mu \mathrm{m}$ compr., 11-28 $\mu \mathrm{m}$ larg., $\mathrm{Rc} / 1=2,2-3,0$; vista polar circular, pólo anterior gradativamente atenuado em colarinho cilíndrico, levemente reto, bordo liso ou crenulado, 2,8-4,6 $\mu \mathrm{m}$ alt. e 4,6-6,5 $\mu \mathrm{m}$ larg.; pólo posterior gradativamente atenuado em processo caudal cônico, reto até $7,5 \mu \mathrm{m}$ compr.; parede granulosa, amarelado-claro; cloropastos numerosos, discóides; grãos de paramido numerosos, bastoniformes; com ou sem pirenóides internos.

Material examinado: HAS 26079, HAS 26327, HAS 26333, HAS 26342, HAS 34654, HAS 34657 , HAS 34712, HAS 34803, HAS 34822, HAS 34831, HAS 34883, HAS 34885, HAS 34887, HAS 34891, HAS 34908, HAS 34910, HAS 34920, HAS 34922, HAS 34965, HAS 34992, HAS 34994, HAS 34998.

Distribuição no Brasil: Rio Grande do Sul (Alvesda-Silva 1998; Franceschini 1992). Rondônia (Albuquerque \& Menezes 1997). São Paulo (Xavier 1993).

Distribuição geográfica: cosmopolita.

11. Strombomonas fluviatilis (Lemm.) Defl. var. levis (Lemm.) Skv., Aus der Biol. Sungari Station zur Harbin 1(2): 78, pl. 6, fig. 33. 1925.

Fig. 20.


$\mathrm{Rc} / \mathrm{l}=2,5-2,7$; pólo anterior alargado, terminando em colarinho de 6,4-7,5 $\mu \mathrm{m}$ alt. e 7,5-9,0 $\mu \mathrm{m}$ larg.; bordo crenulado; pólo posterior acuminado abruptamente em processo caudal cônico, $18-20 \mu \mathrm{m}$ compr.; parede amarelada; protoplasto com estrias levógiras; cloroplastos numerosos, discóides, 3,7-4,6 $\mu \mathrm{m}$ diâm., grãos de paramido numerosos, arredondados ou bastoniformes. Material examinado: HAS 26327, HAS 26342.

Distribuição no Brasil: Rio Grande do Sul (Franceschini 1992; Alves-da-Silva \& Torres 1994).

Distribuição geográfica: cosmopolita.

12. Strombomonas fluviatilis (Lemm.) Defl. var. major Conf., Rev. Hydrobiol. Trop. 26(3): 192 pl. 4, fig. 69-c. 1993.

Fig. 21.

Lórica elíptica 79-85 $\mu \mathrm{m}$ compr., 30-35 $\mu \mathrm{m}$ larg., $\mathrm{Rc} / \mathrm{l}=2,2-2,5$; pólo anterior alargado, terminando em


crenulado; pólo posterior acuminado abruptamente em processo caudal cônico, quase reto, $21-24 \mu \mathrm{m}$ compr.; parede rugosa, castanha-escura; cloroplastos numerosos, discóides, 3,7-4,6 $\mu \mathrm{m}$ diâm.; grãos de paramido numerosos, arredondados ou bastoniformes; flagelo $1 / 8$ a 1/10 do comprimento lórica. 
Material examinado: HAS 34822, HAS 34908.

Distribuição no Brasil: Amazonas (Conforti 1993).

Distribuição geográfica: variedade encontrada exclusivamente na América do Sul.

Comentários: os exemplares encontrados no Parque tem dimensões intermediárias entre $S$. fluviatilis var. major Conforti e $S$. fluviatilis var. rugosa f. major Yacubson. Apesar de apresentar maiores dimensões que a var. major, os indivíduos analisados possuem morfologia e Rc/l mais próxima desta variedade.

Segunda citação da variedade para o país e primeira para o Estado.

13. Strombomonas globulosa Conf. \& Joo, Cryptogamie Algol. 15(4): 274, fig. 66a-b. 1994.

Fig. 22-23.

Lórica elíptica, alargada na região mediana, ca. $20,3 \mu \mathrm{m}$ compr.; ca. $12 \mu \mathrm{m}$ larg.; $\mathrm{Rc} / 1=1,7$; vista polar circular; pólo anterior gradativamente atenuado em colarinho cilíndrico, curto, margem distal reta, bordo liso ou crenulado, ca. $2 \mu \mathrm{m}$ alt.; pólo posterior gradativamente atenuado em processo caudal cônico, reto, curto, ca. $3 \mu \mathrm{m}$ compr.; parede castanho-clara a castanha-avermelhado; cloropastos numerosos, discóides, ca. 1,5 $\mu$ m diâm.; pirenóides nus.

Material examinado: HAS 26342, HAS 34647, HAS 34908.

Distribuição no Brasil: Amazonas (Conforti 1993). Rio Grande do Sul (Alves-da-Silva 1998).

Distribuição geográfica: espécie encontrada na América do Sul e Norte.

14. Strombomonas gibberosa (Playf.) Defl., Arch. Protistenk. 69(3): 595, fig. 97-103. 1930.

Fig. 24-25.

Lórica rombóide, região mediana alargada e mais


$\mathrm{Rc} / \mathrm{l}=2,0-2,4$; vista polar circular; pólo anterior atenuando abruptamente em colarinho cilíndrico, levemente dilatado na extremidade, 9,2-14,5 $\mu \mathrm{m}$ alt. e 5,6-10 $\mu \mathrm{m}$ larg.; pólo posterior atenuado rapidamente em processo caudal cônico de $12-23 \mu \mathrm{m}$ compr.; parede hialina a castanha-clara; cloroplastos numerosos, discóides, ca. 4,0 $\mu$ m diâm.

Material examinado: HAS 26327, HAS 26342, HAS 34632, HAS 34667, HAS 34715, HAS 34793 , HAS 34801, HAS 34805, HAS 34807, HAS 34809 , HAS 34813, HAS 34815, HAS 34817, HAS 34819 , HAS 34822, HAS 34825, HAS 34881, HAS 34885 , HAS 34893, HAS 34908, HAS 34914, HAS 34922,
HAS 34924, HAS 34955, HAS 34957, HAS 34963 , HAS 34965, HAS 34992, HAS 34994, HAS 34998.

Distribuição no Brasil: Amazonas (Uherkovich Schmidt 1974; Conforti 1993). Paraná (Jati \& Train 1994). Rio Grande do Sul (Franceschini 1992; Alvesda-Silva \& Torres 1994; Alves-da-Silva \& Crossetti 1999). Rio de Janeiro (Huzsar 1986).

Distribuição geográfica: cosmopolita.

Comentários: no presente estudo foram encontradas duas populações em diferentes ambientes: 1) população com menores dimensões, $44,4-52 \times 21-22 \mu \mathrm{m}$, com morfologia geral da variedade típica, mas com colarinho mais longo, 9,2-11 $\mu \mathrm{m}$ compr.; 2) população com maiores dimensões, $66-74 \times 28-32 \mu \mathrm{m}$ e colarinho de $9,2-14,5 \mu \mathrm{m}$ compr. A presença de um colarinho com maiores dimensões poderia levar a identificar os indivíduos como $S$. gibberosa var. longicollis. Entretanto, Deflandre (1930) deu dimensões do colarinho de $18 \mu \mathrm{m}$ compr. para esta variedade.

A espécie foi identificada como a típica, com base em Deflandre (1930), Conforti (1993), Starmach (1983) e Tell \& Conforti (1986).

Nevo \& Wasser (2000) citaram a espécie como ocorrendo no plâncton, em corpos d'água efêmeros, rios, lagos, açudes e pântanos, em águas moderadamente poluídas por matéria orgânica. No Parque a espécie foi encontrada em águas com concentração de matéria orgânica entre $1,8-9,5 \mathrm{mg} . \mathrm{L}^{-1}$, em canais, margens de ilhas, foz de rios e sacos.

15. Strombomonas girardiana (Playf.) Defl., Arch. Protistenk. 69(3): 573, fig. 33-35. 1930.

Fig. 26-27.

Lórica sub-hexagonal, lados quase paralelos, levemente aconcavados na região mediana, $40-47 \mu \mathrm{m}$ compr., 20-22 $\mu \mathrm{m}$ larg., $\mathrm{Rc} / 1=2,0-2,3$; vista polar circular; pólo anterior terminando em colarinho curto,

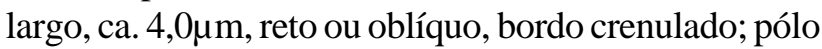
posterior atenuado em processo caudal cônico, reto, ca. $14 \mu \mathrm{m}$ compr.; parede granulosa, hialina a castanhoclara; cloroplastos arredondados ou alongados, ca. de 4,5 $\mu \mathrm{m}$ diâm.; pirenóides internos presentes.

Material examinado: HAS 26327, HAS 26333, HAS 26342, HAS 26347/48, HAS 34632, HAS 34635 , HAS 34647, HAS 34651, HAS 34665, HAS 34667 , HAS 34715, HAS 34791, HAS 34805, HAS 34807 , HAS 34809, HAS 34822, HAS 34881, HAS 34883, HAS 34885, HAS 34908, HAS 34910, HAS 34914, HAS 34922, HAS 34947, HAS 34990, HAS 34992 , HAS 34994, HAS 34998. 
Distribuição no Brasil: Amazonas (Conforti 1993). Paraná (Jati \& Train 1994; Rodrigues 1998). Rio Grande do Sul (Franceschini 1992; Alves-da-Silva \& Torres 1994).

Distribuição geográfica: cosmopolita.

16. Strombomonas lanceolata (Playf.) Defl., Arch. Protistenk. 69(3): 582, fig. 59-60. 1930.

Lórica amplamente elíptica 29-30 $\mu \mathrm{m}$ compr., 11-12 $\mu \mathrm{m}$ larg.; $\mathrm{Rc} / \mathrm{l}=2,5-2,6$; pólo anterior terminando em colarinho curto, largo, levemente oblíquo, com bordo liso, pólo posterior atenuado em processo caudal cônico, reto, ca. $4 \mu \mathrm{m}$ compr.; parede lisa, verde-amarelada; cloroplastos discóides, ca. $3 \mu \mathrm{m}$ diâm.; pirenóides internos presentes.

Material examinado: HAS 26333, HAS 34647, HAS 34813, HAS 34908, HAS 34922, HAS 34992,



Figuras 13-27. 13. Strombomonas cuneata (Playf.) Defl. 14. Strombomonas deflandrei (Roll) Defl. 15-16. Strombomonas ensifera (Daday) Defl. 17-19. Strombomonas fluviatilis (Lemm.) Defl. 20. Strombomonas fluviatilis (Lemm.) Defl. var. levis (Lemm.) Skv. 21. Strombomonas fluviatilis (Lemm.) Defl. var. major Conf. 22-23. Strombomonas globulosa Conf. \& Joo. 24-25. Strombomonas gibberosa (Playf.) Defl. 26-27. Strombomonas girardiana (Playf.) Defl. Escala $=10 \mu \mathrm{m}$.
HAS 34994, HAS 34996, HAS 34998.

Distribuição no Brasil: Amazonas (Conforti 1993). Rio Grande do Sul (Alves-da-Silva \& Torres 1994; Alves-da-Silva \& Avila 1997).

Distribuição geográfica: América do Sul, Ásia, Europa, Oceania.

17. Strombomonas maxima (Skv.) Defl., Arch. Protistenk. 69(3): 583, fig. 61-65. 1930.

Lórica elíptica, 72,1-98 $\mu \mathrm{m}$ compr., 27-41 $\mu \mathrm{m}$ larg., $\mathrm{Rc} / \mathrm{l}=$ 2,3-2,6; pólo anterior terminado em colarinho cilíndrico; truncado, ligeiramente oblíquo, até 7,4 $\mu \mathrm{m}$ alt. e 7,4-9,2 $\mu \mathrm{m}$ larg.; pólo posterior abruptamente atenuado em processo caudal cônico, reto a ligeiramente curvo 18,5-30 $\mu$ m compr.; parede lisa, amarelada-clara, verde a castanha-clara, flexível formando pregas longitudinais e transversais; cloroplastos numerosos, elípticos, ca. $4 \mu \mathrm{m}$ diâm.; pirenóides internos presentes; grãos de paramido numerosos, bastoniformes.

Material examinado: HAS 34647, HAS 34807, HAS 34811, HAS 34822, HAS 34881, HAS 34885 , HAS 34908, HAS 34910, HAS 34922, HAS 34994, HAS 34998.

Distribuição no Brasil: Acre (Keppeler et al. 2000). Mato Grosso (Menezes \& Fernandes 1987; 1989). Rio Grande do Sul (Franceschini 1992; Alvesda-Silva \& Torres 1994; Alves-da-Silva \& Ávila 1997). Rio de Janeiro (Menezes 1994).

Distribuição geográfica: cosmopolita.

18. Strombomonas morenensis Bal. \& Dast., Physis 12: 354.1938.

Fig. 28.

Lórica trapezoidal, 60-62 $\mu \mathrm{m}$ compr., 30-31 $\mu \mathrm{m}$ larg., $\mathrm{Rc} / 1=2,0$; pólo anterior estreita-se abruptamente terminando em colarinho longo, cilíndrico, 10-11 $\mu \mathrm{m}$ compr., com bordo crenulado levemente oblíquo, ca. $8 \mu \mathrm{m}$ larg.; pólo posterior atenua-se abruptamente em processo caudal 16-18,5 $\mu \mathrm{m}$ compr.; parede rugosa, castanho-clara, finamente pontuada; cloroplastos numerosos, discóides, 3,5-4,0 $\mathrm{um}$ diâmetro.

Material examinado: HAS 26079, HAS 34811.

Distribuição no Brasil: nova citação para o Estado e para o país.

Distribuição geográfica: espécie encontrada exclusivamente na América do Sul.

Comentários: os exemplares encontrados se apresentaram ligeiramente menores que os indivíduos encontrados na Argentina, principalmente o colarinho e o processo caudal. Balech \& Dastugue (1938) citam colarinho com comprimento até $19 \mu \mathrm{m}$ e o processo 
caudal de $19-20 \mu \mathrm{m}$, enquanto os exemplares do Parque apresentaram-se com colarinho até $11 \mu \mathrm{m}$ e processo caudal até $18,5 \mu \mathrm{m}$ compr.

19. Strombomonas napiformis (Playf.) Defl. var. brevicollis Playf., Arch. Protistenk. 69(3): 591-592, fig. 83-84. 1930.

Fig. 29-30.

Lórica obotriangular a obovada, 44,4-51 $\mu \mathrm{m}$ compr., 24-26 $\mu \mathrm{m}$ larg., Rc/1 = 1,8-2,0; pólo anterior mais alargado que o posterior, terminando rapidamente em colarinho cilíndrico, curto, 3,7-4,6 $\mu$ m compr., com bordo alargado levemente oblíquo, 7,4-8,3 $\mu \mathrm{m}$ larg.; pólo posterior estreita-se abruptamente em um processo caudal longo, 9,2-12 $\mu \mathrm{m}$ compr.; parede levemente granulosa, hialina a amarelada; paramidos numerosos, bastoniformes ca. $1,5 \mu \mathrm{m}$ compr.; ca. 8 pirenóides internos.

Material examinado: HAS 34720, HAS 34831.

Distribuição no Brasil: nova citação para o Estado e para o país.

Distribuição geográfica: Ásia, Oceania.

Comentários: difere da variedade tipo pela forma mais obovada, pelo processo caudal mais longo e pelo bordo do colarinho largo e inclinado.

Segundo Deflandre (1930) esta variedade possui ca. 12 cloroplastos grandes, poliédricos, arredondados. No material examinado encontrou-se mais de 8 cloroplastos com pirenóides internos, que vistos de cima dão a impressão descrita pelo autor da variedade de cloroplastos grandes e arredondados. A presença de pirenóides internos, ora encontrados nos indivíduos estudados, vem contribuir na ampliação do conhecimento da variedade.

20. Strombomonas rotunda (Playf.) Defl., Arch. Protistenk. 69(3): 593, fig. 88, 1930.

Fig. 31-34.

Lórica obovada, com região mediana transversalmente elíptica, 27-31 $\mu \mathrm{m}$ compr., 17-18 $\mu \mathrm{m}$ larg., $\mathrm{Rc} / 1=1,6-1,7$; vista polar circular; pólo anterior atenuado abruptamente em colarinho cilíndrico, com bordo reto ou levemente oblíquo, liso ou crenulado, com 5-6,4 $\mu \mathrm{m}$ alt. e 6-7,0 $\mu \mathrm{m}$ larg.; pólo posterior abruptamente atenuado em processo caudal agudo, reto, de 6-10 $\mu \mathrm{m}$ compr.; parede rugosa, castanho-clara a avermelhada; cloroplastos numerosos 5-6 discóides, ca. $5 \mu \mathrm{m}$ diâm.; pirenóides nus; grãos de paramido numerosos, elípticos ca. $3 \mu \mathrm{m}$ diâm.; flagelo ca. uma vez o comprimento da lórica.

Material examinado: HAS 26327, HAS 26333,
HAS 34654, HAS 34657, HAS 34881, HAS 34994 , HAS 34998.

Distribuição no Brasil: Amazonas (Uherkovich \& Rai 1979). Rio Grande do Sul (Alves-da-Silva \& Ferraz 1991; Alves-da-Silva et al. 1994; Alves-da-Silva \& Torres 1994). Rio de Janeiro (Menezes 1994). São Paulo (Xavier 1985; 1988; 1993).

Distribuição geográfica: América do Norte, América do Sul, Ásia, Austrália, Oceania.

Comentários: Deflandre (1930) descreve esta espécie com a região mediana da lórica transversalmente elíptica, pólo anterior atenuado em colarinho reto, largo, com aproximadamente $8 \mu \mathrm{m}$ compr. e processo caudal obtuso com $10 \mu \mathrm{m}$ compr.

No Parque, os espécimens encontrados concordam quanto à morfologia com a descrição da espécie, diferindo, entretanto, no comprimento do colarinho que se apresentou no máximo com ca. $6,4 \mu \mathrm{m}$ compr. e no processo caudal menor, alguns com apenas $6 \mu \mathrm{m}$ compr. Os resultados aqui encontrados corroboram aqueles registrados por Menezes (1994) em ambientes do Rio de Janeiro, cujos espécimes apresentaram também menor altura do colarinho (até $5 \mu \mathrm{m}$ compr.) e processo caudal, no máximo, com $7 \mu \mathrm{m}$ comprimento.

21. Strombomonas scabra (Playf.) Tell \& Conf. var. coronata Conf. \& Joo, Cryptogamie Algol. 15(4): 277, fig. 58-59a-d. 1994.

Lórica ovado-elíptica, ca. $22,2 \mu \mathrm{m}$ compr., ca. $18,5 \mu \mathrm{m}$ larg., $\mathrm{Rc} / \mathrm{l}=1,2$; pólo anterior atenuado abruptamente em colarinho em forma de coroa, formado por projeções irregulares, margem distal divergente; pólo posterior levemente acuminado em processo caudal ca. 1,0 $\mu \mathrm{m}$ compr.; parede espessa, granulosa, castanho-avermelhada; cloropastos numerosos, discóides, ca. 2,0 $\mu$ m diâm.

Material examinado: HAS 34996.

Distribuição no Brasil: Rio Grande do Sul ( Alvesda-Silva \& Ávila 1997).

Distribuição geográfica: América do Norte, América do Sul.

Comentários: difere da variedade típica da espécie pelo colarinho em forma de coroa.

Os exemplares examinados apresentaram dimensão do processo caudal inferior as dadas pelos autores da variedade, mas concordam com a fig. 58d de Conforti \& Joo (1994). Não foi observado, também, a presença de pontuações na lórica, entretanto, o colarinho terminando em forma de coroa com margem distal divergente concorda com a descrição da variedade.

Segunda citação para o Estado e para o país. 
22. Strombomonas scabra (Playf.) Tell \& Conf. var. intermedia (Yacubson) Tell \& Conf., Nova Hedwigia 46(3-4): 545, pl. 6, fig. 13-16. 1988.

Fig. 35 .

Lórica elíptica, 41-45 $\mu \mathrm{m}$ compr., 18,5-21 $\mu \mathrm{m}$ larg., $\mathrm{Rc} / 1=2,1-2,2$; vista polar circular; pólo anterior levemente atenuado, terminando em colarinho alto, cilíndrico, bordo crenulado 5,5-6,5 $\mu \mathrm{m}$ alt. e 4-5,5 $\mu \mathrm{m}$ larg.; pólo posterior atenuado em processo caudal curto arredondado a truncado no máximo $3 \mu \mathrm{m}$ compr.; parede espessa, granulosa, com dobras, castanho-claro a castanho-avermelhada; cloropastos numerosos, discóides, ca. 2,5um diâm.; pirenóides internos.

Material examinado: HAS 26327, HAS 26333, HAS 34805, HAS 34819, HAS 34881, HAS 34922 , HAS 34990, HAS 34994.

Distribuição no Brasil: Amazonas (Conforti 1993). Rio Grande do Sul (Alves-da-Silva et al. 1991; Alvesda-Silva \& Torres 1994; Alves-da-Silva 1998).

Distribuição geográfica: variedade encontrada exclusivamente na América do Sul.

Comentários: foram observados alguns exemplares com pirenóides internos, ainda não registrados em literatura para a variedade em questão.

23. Strombomonas scabra (Playf.) Tell \& Conf. var. labiata (Hub.-Pest.) Tell \& Conf., Nova Hedwigia. 46(3-4): 545-546, pl. 6, fig. 4-5. 1988.

Lórica ovada a elíptica, ca. de $22,2 \mu \mathrm{m}$ compr., 16,2-18,5 $\mu \mathrm{m}$ larg., $\mathrm{Rc} / \mathrm{l}=1,3$; pólo anterior terminando em um colarinho bem diferenciado, ca. $4 \mu \mathrm{m}$ alt., e ca. $6,5 \mu \mathrm{m}$ larg., pólo posterior atenuado ligeiramente em um processo caudal ca. $1,8 \mu \mathrm{m}$ compr.; parede granulosa, castanho-avermelhado; cloroplastos discóides, ca. $3 \mu \mathrm{m}$ diâm.

Material examinado: HAS 34990, HAS 34994, HAS 34996.

Distribuição no Brasil: Amazonas (Conforti 1993). Rio Grande do Sul (Alves-da-Silva \& Ávila 1997).

Distribuição geográfica: América do Sul, Europa.

24. Strombomonas scabra (Playf.) Tell \& Conf. var. longicollis (Playf.) Tell \& Conf., Nova Hedwigia 46(3-4): 546, pl. 2, fig. 4, pl. 7, fig. 1. 1988.

Fig. 36.


$\mathrm{Rc} / 1=1,4-1,5 ;$ pólo anterior terminando em um colarinho bem diferenciado 3,5-4,5 $\mu \mathrm{m}$ alt. e 4,5-5,5 $\mu \mathrm{m}$ larg.; pólo posterior arredondado; parede granulosa, amarela a castanho-avermelhado; cloroplastos discóides ca. $3 \mu \mathrm{m}$ diâmetro.
Material examinado: HAS 26070, HAS 26333, HAS 34805.

Distribuição no Brasil: Amazonas (Conforti 1993). Mato Grosso (Menezes \& Fernandes 1987). Rio Grande do Sul (Alves-da-Silva \& Torres 1994).

Distribuição geográfica: América do Sul, Europa, Oceania.

Comentários: difere da variedade típica da espécie pela presença de colarinho alto, cilíndrico, reto ou levemente oblíquo, com bordo crenulado, 3,5-4,5 $\mu \mathrm{m}$ comprimento.

25. Strombomonas scabra (Playf.) Tell \& Conf. var. ovata (Playf.) Tell \& Conf. f. minor Tell \& Conf., Nova Hedwigia 46(3-4): 546, pl. 2, fig. 2-3, pl. 6, fig. 11-12. 1988.

Fig. 39-41.

Lórica elíptica, alargada na região anterior, ca. $18,2 \mu \mathrm{m}$ compr., ca. $14,4 \mu \mathrm{m}$ larg., $\mathrm{Rc} / \mathrm{l}=1,3$; vista polar circular; pólo anterior levemente atenuado, terminando em colarinho cilíndrico, levemente curto, bordo crenulado 2,5-3,5 $\mu \mathrm{m}$ alt. e $2 \mu \mathrm{m}$ larg.; pólo posterior acuminado; parede espessa, granulosa; cloropastos numerosos, discóides, ca. 1,5 $\mu \mathrm{m}$ diâmetro.

Material examinado: HAS 34632, HAS 34635, HAS 34793, HAS 34801, HAS 34883, HAS 34889, HAS 34891, HAS 34908, HAS 34922, HAS 34947, HAS 34969, HAS 34990.

Distribuição no Brasil: Amazonas (Conforti 1993). Rio Grande do Sul (Alves-da-Silva 1998).

Distribuição geográfica: variedade encontrada exclusivamente na América do Sul.

Comentários: difere da variedade típica pelas menores dimensões da lórica. Os indivíduos se apresentaram ligeiramente mais largos que a dimensão dada na descrição original.

26. Strombomonas scabra (Playf.) Tell \& Conf. var. ovata (Playf.) Tell \& Conf. f. ovata (Defl.) Tell \& Conf., Nova Hedwigia 46(3-4): 546, pl. 2, fig. 5-6, pl.6, fig. 8-10. 1988.

Fig. 37-38.

Lórica elíptica a ovada, 30-32,4 $\mu \mathrm{m}$ compr., 18,5-21,3 $\mu \mathrm{m}$ larg., Rc/l = 1,4-1,7; pólo anterior abruptamente atenuado, terminando em colarinho alto, com bordo crenulado com ca. $4 \mu \mathrm{m}$ compr. e 3,5 $\mu \mathrm{m}$ larg.; pólo posterior acuminado, terminando em processo caudal curto com até $3 \mu \mathrm{m}$ compr.; parede espessa, escabrosa, castanho-claro a escura; cloropastos numerosos, discóides, até ca. 2,5 $\mu \mathrm{m}$ diâm.; duplo- 
pirenóides presentes; flagelo até duas vezes o comprimento da lórica.

Material examinado: HAS 26327, HAS 34651, HAS 34654, HAS 34657, HAS 34667, HAS 34805, HAS 34825, HAS 34881, HAS 34914, HAS 34922, HAS 34992, HAS 34994, HAS 34998.

Distribuição no Brasil: Rio Grande do Sul (Alvesda-Silva \& Torres 1994).

Distribuição geográfica: América do Sul, Oceania.

Comentários: difere da variedade típica da espécie por sua lórica elíptica a ovada e por apresentar o pólo posterior levemente atenuado em processo caudal com até $3 \mu \mathrm{m}$ comprimento.

27. Strombomonas scabra (Playf.) Tell \& Conf. var. scabra, Nova Hedwigia 46(3-4): 545, pl. 1, fig. 1-6, pl. 6, fig. 1-3. 1988.

Fig. 42-43.

Lórica amplamente elíptica a globosa, 18-26 $\mu \mathrm{m}$ compr., 15-21,3 $\mu \mathrm{m}$ larg., $\mathrm{Rc} / 1=1,2$; pólo anterior abruptamente atenuado, terminando em colarinho curto, baixo, bordo crenulado ca. $1,5 \mu \mathrm{m}$ alt. e 5,5 $\mu \mathrm{m}$ larg.; pólo posterior arredondado, sem processo caudal; parede espessa, granulosa a escabrosa, castanho-claro a escura; cloropastos numerosos, discóides, até ca. $3 \mu \mathrm{m}$ diâm.; pirenóides internos; flagelo até duas vezes o comprimento da lórica.

Material examinado: HAS 26087, HAS 26333, HAS 26342, HAS 34635, HAS 34637, HAS 34643, HAS 34654 , HAS 34715, HAS 34791, HAS 34795, HAS 34799, HAS 34801, HAS 34805, HAS 34807, HAS 34813, HAS 34815, HAS 34817, HAS 34881, HAS 34883, HAS 34885, HAS 34893, HAS 34947, HAS 34953, HAS 34957, HAS 34990, HAS 34996, HAS 34998.

Distribuição no Brasil: Mato Grosso (Menezes \& Fernandes 1987; 1989). Rio Grande do Sul (Alves-daSilva \& Torres 1994; Alves-da-Silva 1998). Rio de Janeiro (Marinho et al. 1993). São Paulo (Bicudo et al. 1999).

Distribuição geográfica: cosmopolita.

28. Strombomonas schauinslandii (Lemm.) Defl., Arch. Protistenk. 69(3): 594, fig. 90-96. 1930.


larg., $\mathrm{Rc} / 1=1,6-1,9$; vista polar arredondada; pólo anterior terminado em colarinho cilíndrico com extremidade distal levemente divergente 4,5-5,5 $\mu \mathrm{m}$ alt. e 5,5-8,3 $\mu \mathrm{m}$ larg.; pólo posterior atenuado em processo caudal cônico, 8-11 $\mu \mathrm{m}$ compr.; parede granulosa, castanho-clara; cloroplastos numerosos, discóides, ca. $2 \mu \mathrm{m}$ diâm.; com ou sem pirenóides internos.
Material examinado: HAS 26327, HAS 34908, HAS 34992, HAS 34994.

Distribuição no Brasil: Amazonas (Conforti 1993). Rio Grande do Sul (Alves-da-Silva \& Crossetti 1999).

Distribuição geográfica: cosmopolita.

Comentários: segundo Tell \& Conforti (1986), os exemplares encontrados em região subtropical da Argentina apresentaram menores dimensões $(18 \mu \mathrm{m}$ compr. $\times 10 \mu \mathrm{m}$ larg.), fato não observado no material analisado no Parque.

29. Strombomonas tetraptera Bal. et Dast. var. gallica Bour. \& Coute, Rev. Algol. 13(4): 299, fig. 17. 1978.

Fig. 44-47.

Lórica de contorno geral elíptica turbinada, ângulos proeminentes formando projeções aliformes, 32,3-39 $\mu \mathrm{m}$ compr., 20,3-22,2 $\mu \mathrm{m}$ larg., Rc/l = 1,5-1,8; vista polar quadrangular, lados côncavos, ângulos arredondados; pólo anterior alongado, atenuado em colo cilíndrico, curto, levemente oblíquo, 1,8-2,8 $\mu$ m compr. e 8,3-9,2 $\mu \mathrm{m}$ larg.; pólo posterior atenuado abruptamente em processo caudal muito curto, 2,8-4,6 $\mu \mathrm{m}$ compr., parede rugosa ou granulosa, amarelo-esverdeada a verdeclara; cloroplastos numerosos, discóides ca. $4 \mu \mathrm{m}$ diâm.; pirenóides internos presentes.

Material examinado: HAS 26329, HAS 26333, HAS 26347, HAS 26348.

Distribuição no Brasil: Mato Grosso (Menezes \& Fernandes 1987, como S. tetraptera).

Distribuição geográfica: Ásia, França.

Comentários: segundo Bourrelly \& Coute (1978), esta variedade difere da espécie típica pela torção regular helicóide da lórica, pelas menores dimensões e pelo vista polar com ângulos arredondados, sem expansões aliformes. Os exemplares apresentaram-se ligeiramente maiores em largura que o citado para o material francês (Glareins, Ain, região de Dambes).

Considera-se que o material encontrado por Menezes e Fernandes (1987) no município de Cáceres, no Mato Grosso, trata-se da var. gallica e não a espécie típica.

Os indivíduos desta variedade foram encontrados em temperatura entre $28-30^{\circ} \mathrm{C}$.

Nova citação de ocorrência da espécie para Estado e segunda para o país.

30. Strombomonas treubii (Wol.) Defl., Arch. Protistenk. 69(3): 592-593, fig. 86. 1930

Fig. 48-49.

Lórica elíptica, com região mediana alargada, 
39,7-42 $\mu$ m compr., 19,4-21 $\mu \mathrm{m}$ larg., $\mathrm{Rc} / \mathrm{l}=2,0$; vista polar arredondada; pólo anterior atenuando rapidamente em um colarinho reto à levemente oblíquo,


levemente crenulado; pólo posterior atenuando abruptamente em processo caudal cônico, hialino de 13-14 $\mu \mathrm{m}$ compr.; parede granulosa ou rugosa, acastanhada a levemente avermelhada; cloroplastos numerosos, discóides ca. 3,0 $\mu \mathrm{m}$ diâm.; sem ou com pirenóides internos presentes; flagelo ca. $1 / 3 \mu \mathrm{m}$ o comprimento da lórica.

Material examinado: HAS 26327, HAS 26333, HAS 26339, HAS 26342, HAS 34637, HAS 34643, HAS 34654, HAS 34657, HAS 34799, HAS 34801, HAS 34811, HAS 34813, HAS 34815, HAS 34817 , HAS 34822, HAS 34831, HAS 34953, HAS 34959, HAS 34994, HAS 34996. 1992).

Distribuição no Brasil: Rio de Janeiro (Menezes

Distribuição geográfica: América do Sul, Ásia.

Comentários: os espécimens encontrados são semelhantes quanto a morfologia a $S$ rotunda, diferindo desta última por apresentarem maiores dimensões celulares, célula levemente mais assimétrica e maior relação comprimento $\times$ largura. Segundo Deflandre (1930), pode existir superposição de medidas entre estas duas espécies.

Tell \& Conforti (1986) mencionaram que S. treubii possui colarinho reto ou infundibiliforme com seção oblíqua, o que concorda com os espécimes registrados para o Parque.

Nova citação de ocorrência da espécie para Estado e segunda para o país.

31. Strombomonas triquetra (Playf.) Defl. var. torta Rino, Rev. Ciênc. Biol. 5: 169, pl. 9, fig. 7-10. 1972.

Lórica levemente triangular a trapezoidal,

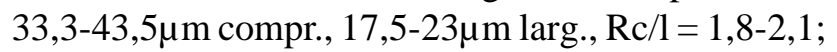
vista polar triangular com ângulos arredondados; pólo anterior abruptamente atenuado em colarinho truncado, oblíquo, largo, bordo crenulado ca. $4 \mu \mathrm{m}$ alt., e 6-7 $\mu \mathrm{m}$ larg.; pólo posterior abruptamente atenuado em processo caudal cônico 3-4,5 $\mu$ m compr.; parede rugosa ou granulosa, castanho-claro a castanhomédio, com dobras principalmente na região anterior e pregas irregulares, lados ondulados, constritos; cloropastos numerosos, discóides, $3 \mu \mathrm{m}$ diâm.; pirenóides internos presentes; flagelo uma vez o comprimento da lórica.

Material examinado: HAS 26079, HAS 26327, HAS 26333, HAS 34647, HAS 34654, HAS 34657 ,
HAS 34712, HAS 34715, HAS 34791, HAS 34805 , HAS 34809, HAS 34811, HAS 34813, HAS 34822, HAS 34831, HAS 34885, HAS 34887, HAS 34908, HAS 34969, HAS 34992, HAS 34994, HAS 34996, HAS 34998.

Distribuição no Brasil: Rio Grande do Sul (Alvesda-Silva \& Ávila 1997; Alves-da-Silva 1998).

Distribuição geográfica: África, América do Sul, Ásia.

32. Strombomonas urceolata (Stokes) Defl., Arch. Protistenk. 69(3): 586, fig. 70,71-73. 1930

Fig. 50-51.

Lórica urceolada, 42-51 $\mu \mathrm{m}$ compr., 25-30 $\mu \mathrm{m}$ larg., $\mathrm{Rc} / \mathrm{l}=1,7$; pólo anterior atenuado abruptamente em colarinho curto, subcilíndrico, alargado na região distal, com 2,9-3,7 $\mu \mathrm{m}$ alt. e 6,5-9,5 $\mu \mathrm{m}$ larg.; pólo posterior terminando em processo caudal reto, cônico, 7,4-8,3 $\mu \mathrm{m}$ compr.; parede granulosa, hialina a esverdeada; sem ou com 10-12 pirenóides internos; cloroplastos numerosos, alongados ou discóides de 2-5 $\mu$ m diâmetro.

Material examinado: HAS 26333, HAS 34654, HAS 34657, HAS 34805, HAS 34811.

Distribuição no Brasil: Amazonas (Conforti 1993). Mato Grosso (Menezes \& Fernandes 1987; Menezes 1992).

Distribuição geográfica: cosmopolita.

Comentários: além da presença de cloroplastos grandes e falta de pirenóides citada para esta espécie por Deflandre (1930), foram observados, em alguns indivíduos, pirenóides internos e cloroplastos alongados pequenos.

A presença de pirenóides internos para este táxon amplia o conhecimento da espécie.

Nevo \& Wasser (2000) citaram a espécie como planctônica ocorrendo em corpos d'água efêmeros, açudes, lagos, reservatórios, rios e pântanos e também indicadoras de condições olissapróbicas. No Parque a espécie foi encontrada como planctônica em canal, sacos e margem de ilhas.

33. Strombomonas verrucosa (Daday) Defl. var. verrucosa, Arch. Protistenk. 69, n 3: p. 566, fig. 6-10. 1930.

Lórica elíptica, ovada ou trapezoidal, 35-55,5 $\mu \mathrm{m}$ compr., 15-24,5 $\mu$ m larg., $\mathrm{Rc} / 1=1,9-2,3$; vista polar circular; pólo anterior atenuado gradativamente, em colarinho cilíndrico, reto truncado-oblíquo, bordo liso ou crenulado ca. $4 \mu \mathrm{m}$ alt. e ca. $7 \mu \mathrm{m}$ larg.; pólo posterior atenuado em processo caudal cônico, reto ou levemente curvo, $10-12 \mu \mathrm{m}$ compr.; parede rugosa a granulosa, desde amarelada, acastanhada-clara, acastanhada- 


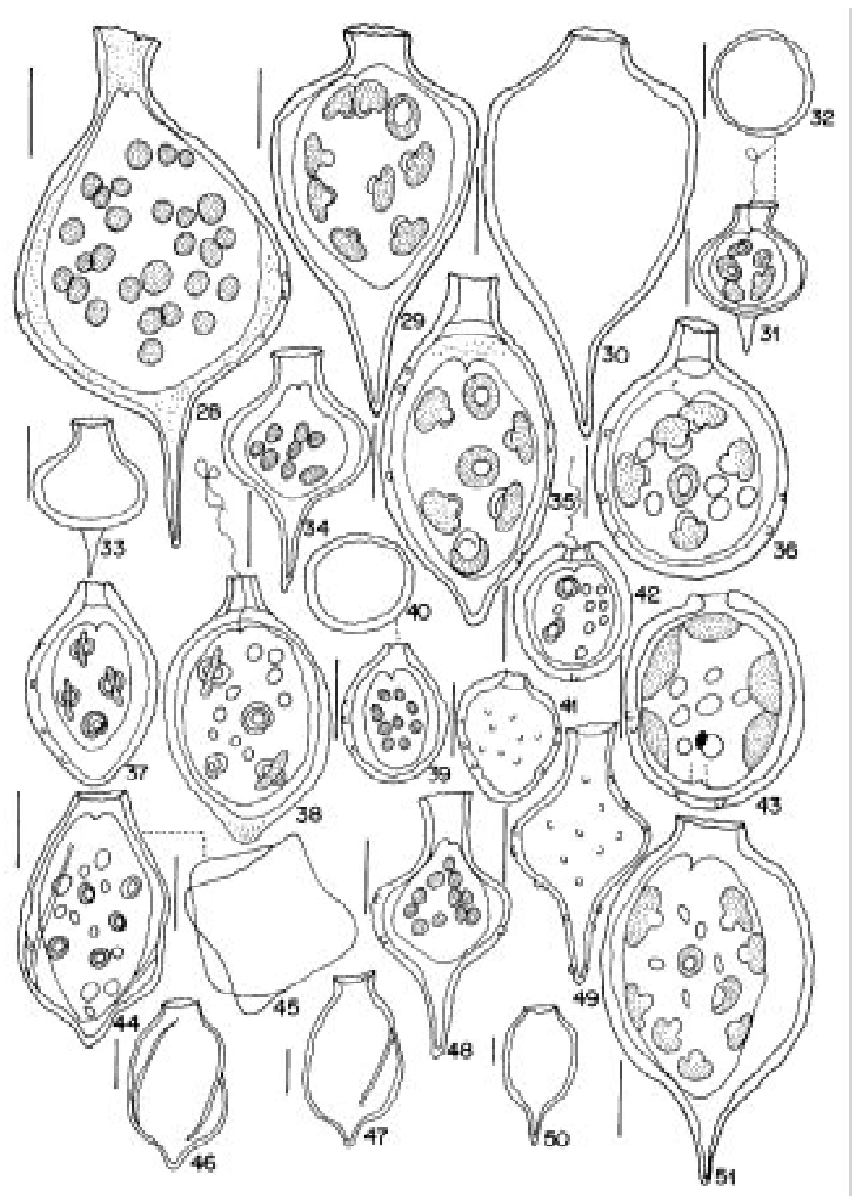

Figuras 28-51. 28. Strombomonas morenensis Bal. \& Dast. 29-30. Strombomonas napiformis (Playf.) Defl. var. brevicollis Playf. 31-34. Strombomonas rotunda (Playf.) Defl. 32. Vista polar. 35. Strombomonas scabra (Playf.) Tell \& Conf. var. intermedia (Yacubson) Tell \& Conf. 36. Strombomonas scabra (Playf.) Tell \& Conf. var. longicollis (Playf.) Tell \& Conf. 37-38. Strombomonas scabra (Playf.) Tell \& Conf. var. ovata (Playf.) Tell \& Conf. f. ovata (Defl.) Tell \& Conf. 39-41. Strombomonas scabra (Playf.) Tell \& Conf. var. ovata (Playf.) Tell \& Conf. f. minor Tell \& Conf. 40. Vista polar. 41. Vista lateral. 42-43. Strombomonas scabra (Playf.) Tell \& Conf. 44-47. Strombomonas tetraptera Bal. et Dast. var. gallica Bour. et Coute. 48-49. Strombomonas treubii (Wol.) Defl. 50-51. Strombomonas urceolata (Stokes) Defl. Escala $=10 \mu \mathrm{m}$.

avermelhada a escura; presença de dobras ou pregas irregulares; cloropastos numerosos, discóides, ca. $4 \mu \mathrm{m}$ diâm.; grãos de paramido numerosos, bastoniformes ou elípticos, ca. $2 \mu \mathrm{m}$ compr.; flagelo ca. uma vez o comprimento celular.

Material examinado: HAS 26081, HAS 26327, HAS 26333, HAS 34632, HAS 34647, HAS 34651, HAS 34654, HAS 34657, HAS 34667, HAS 34709, HAS 34712, HAS 34720, HAS 34791, HAS 34793, HAS 34805, HAS 34807, HAS 34809, HAS 34811, HAS 34813, HAS 34819, HAS 34822, HAS 34825,
HAS 34831, HAS 34881, HAS 34883, HAS 34885, HAS 34887, HAS 34908, HAS 34910, HAS 34914, HAS 34916, HAS 34920, HAS 34922, HAS 34924, HAS 34945, HAS 34947, HAS 34949, HAS 34957 , HAS 34959, HAS 34961, HAS 34963, HAS 34969 , HAS 34990, HAS 34992, HAS 34994, HAS 34996, HAS 34998.

Distribuição no Brasil: Rio Grande do Sul (Alvesda-Silva 1998).

Distribuição geográfica: cosmopolita.

34. Strombomonas verrucosa (Daday) Defl. var. genuina Defl., Arch. Protistenk. 69(3): 565. 1930.

Lórica ovada, 28-30,5 $\mu \mathrm{m}$ compr., 18,5-21 $\mu \mathrm{m}$ larg., $\mathrm{Rc} / 1=1,3-1,6$; vista polar circular; pólo anterior atenuado gradativamente, em colarinho curto, ca. $2 \mu \mathrm{m}$ alt. e ca. $3 \mu \mathrm{m}$ larg.; pólo posterior atenuado abruptamente em processo caudal cônico, arredondado, curto ca. 2,8 $\mu \mathrm{m}$ compr.; parede rugosa a granulosa, acastanhada; cloropastos numerosos, discóides, ca. $3 \mu \mathrm{m}$ diâmetro.

Material examinado: HAS 34651, HAS 34805, HAS 34908, HAS 34963, HAS 34994, HAS 34996.

Distribuição no Brasil: Amazonas (Conforti 1993). Rio Grande do Sul (Alves-da-Silva 1988; Alves-da-Silva \& Ferraz 1991; Alves-da-Silva et al. 1991; Alves-daSilva \& Torres 1994; Alves-da-Silva \& Ávila 1997).

Distribuição geográfica: América do Sul, Ásia, Europa.

Comentários: difere da variedade típica pela presença de pólo posterior atenuado abruptamente em processo caudal cônico, arredondado, curto ca. $2,8 \mu \mathrm{m}$ comprimento.

O gênero Strombomonas esteve muito bem representado no presente estudo, com a presença de 34 táxons específicos e infra-específicos assim distribuídos: 21 espécies, 11 variedades taxonômicas, que não as típicas de suas respectivas espécies e duas formas igualmente não típicas de suas respectivas variedades.

Os táxons mais representativos quanto à ocorrência nos ambientes estudados foram Strombomonas borystheniensis, S. ensifera, S. gibberosa, S. girardiana, S. scabra, S. triquetra var. torta e $S$. verrucosa sendo registrados em mais de $50 \%$ das ambientes amostrados, em especial, S. verrucosa pela ampla distribuição no Parque Estadual Delta do Jacuí, com a presença em $96 \%$ dos locais. É interessante registrar que os táxons em sua maioria ocorreram com baixa densidade relativa. 
Strombomonas elegans, S. lanceolata, S. maxima, S. scabra var. coronata, S. scabra var. labiata, S. shauinslandii, S. triquetra var. torta, $S$. verrucosa e $S$. verrucosa var. genuina são táxons já registrados para a área do Parque por Alves-daSilva \& Ávila (1997) e Alves-da-Silva \& Crossetti (1999).

Strombomonas chodatti, S. cuneata, S. morenensis, S. napiformis var. brevicollis são primeiras citações de ocorrência para o Estado e para o Brasil, sendo $S$. confortii também, novo registro para o Rio Grande do Sul.

São considerados raros por terem ocorrido no máximo em dois locais amostrados: S. fluviatilis var. levis, S. fluviatilis var. major, S. napiformis var. brevicollis, S. scabra var. coronata, S. scabra var. labiata e S. schauinslandii.

Oito táxons possuem distribuição cosmopolita e S. confortii, S. fluviatilis var. major, S. morenensis, S. scabra var. intermedia e S. scabra var. ovata f. minor são citados exclusivamente para a América do Sul.

Os ambientes que mais se destacaram quanto à riqueza do gênero Strombomonas, no Parque Estadual Delta do Jacuí, foram o saco do Cabral (20 táxons), saco do Jacaré (16 táxons), canal das Balseiras e Ilha do Siqueira ambos com 14 táxons (Fig. 52).
Quanto às variáveis ambientais verificou-se que muitos suportaram grande amplitude de $\mathrm{pH}$, mas com maior número de táxons ocorrendo em águas com $\mathrm{pH}$ ácido.

Doze táxons podem ser considerados como euritérmicos, por ocorrerem em amplitude de temperatura superior a $10^{\circ} \mathrm{C}$, enquanto 14 táxons ocorreram em amplitudes menores que $10^{\circ} \mathrm{C}$ (estenotérmicos).

A maioria dos táxons ocorreu em águas com deficiência de oxigênio dissolvido (1,0 a 2,5mg.L $\left.\mathrm{L}^{-1}\right) \mathrm{e}$ em águas com valores de oxigênio dissolvido alto $\left(9,2 \mathrm{mg} \cdot \mathrm{L}^{-1}\right)$.

Observou-se concentrações relativamente altas de nutrientes não havendo limitação de fósforo na forma de ortofosfato, visto que o gênero foi encontrado em águas com $30 \mu \mathrm{g} . \mathrm{L}^{-1}$ até $11.500 \mu \mathrm{g} . \mathrm{L}^{-1}$. Quanto ao amônio e nitrato foram registrados representantes de Strombomonas em águas desde $10 \mu \mathrm{g} . \mathrm{L}^{-1}$ até $1800 \mu \mathrm{g} . \mathrm{L}^{-1}$ e $300 \mu \mathrm{g} . \mathrm{L}^{-1}$ até $2100 \mu \mathrm{g} . \mathrm{L}-1$, respectivamente.

A transparência oscilou nos ambientes estudados entre 10 e $80 \mathrm{~cm}$, mas os táxons foram mais encontrados em águas com valores de transparência até $25 \mathrm{~cm}$.

Quanto à matéria orgânica, o gênero foi encontrado em águas com 1,4mg.L. $\mathrm{L}^{-1}$ até $9,5 \mathrm{mg} . \mathrm{L}^{-1}$, mas com maior

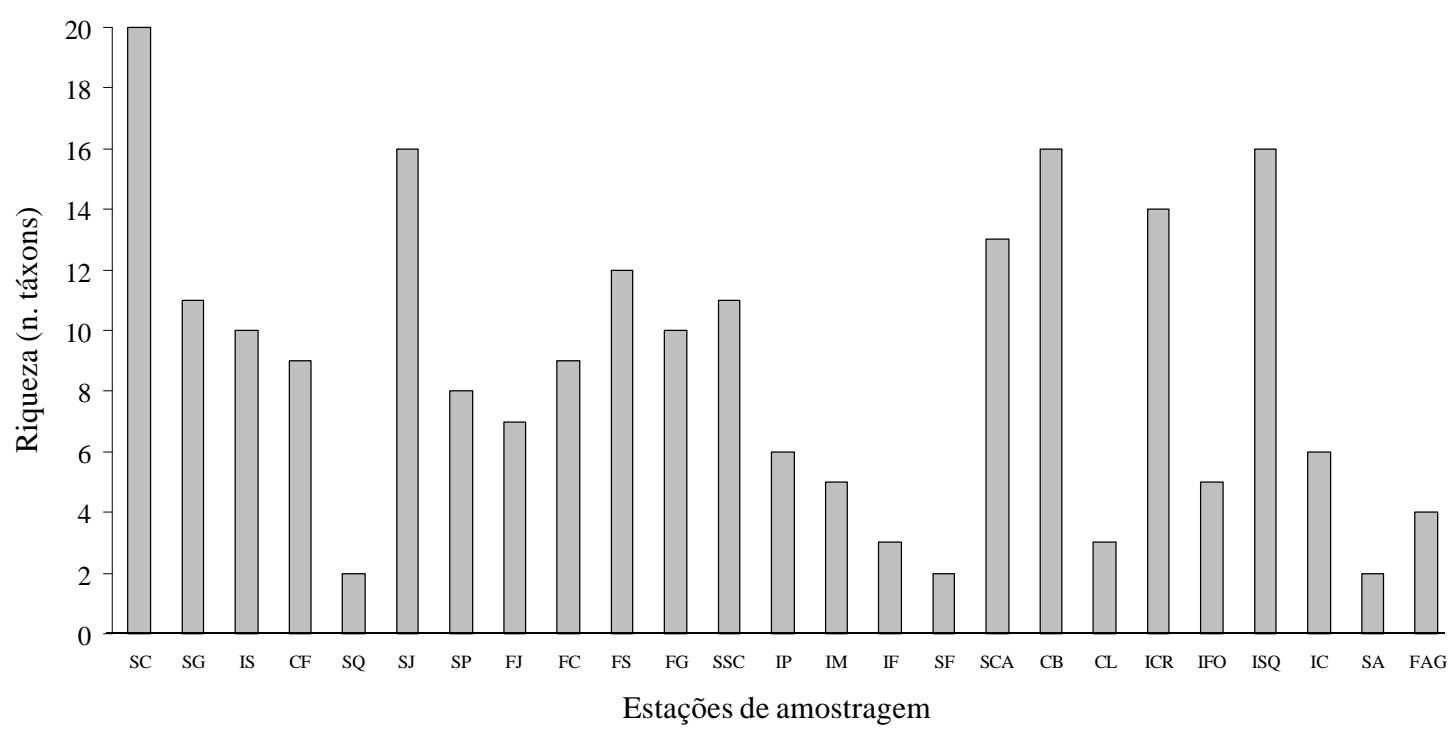

Figura 52. Distribuição do número de táxons do gênero Strombomonas, nas diferentes estações de amostragem, da esquerda para direita: $\mathrm{SC}=$ saco do Cabral, $\mathrm{SG}=$ saco das Garças; IS = ilha do Serafim, CF = canal Feliz; SQ = saco do Quilombo, SJ = saco do Jacaré, SP = saco da Pólvora, FJ = foz do rio Jacuí, FC = foz do rio Caí, FS = foz do rio dos Sinos, FG = foz do rio Gravataí, $\mathrm{SSC}=$ saco Santa Cruz, $\mathrm{IP}=$ ilha das Pombas, IM = ilha dos Marinheiros, IF = ilha das Flores, SF = saco do Ferraz, SCA = saco dos Cachorros, CB = canal das Balseiras, $\mathrm{CL}=$ canal do Lage, ICR = ilha do Cravo, IFO = ilha da Formiga, ISQ = ilha dos Siqueiras, IC = ilha da Cabeçuda, $\mathrm{SA}=\mathrm{saco}$ da Alemoa e FAG = foz do arroio Guaíba. 
número de táxons ocorrendo em águas entre 3 e 5mg. $\mathrm{L}^{-1}$.

Conforti et al. (1994) trabalharam com ultraestrutura e composição química dos elementos da lórica de Trachelomonas e Strombomonas e concluiram por meio de ánalise com microscópio eletrônico e de varredura e microanálises com raio X, que as lóricas de $T$. similis e $T$. volvocina possuiam como primeiro elemento o ferro, enquanto $S$. planctonica e $S$. deflandre o primeiro elemento era a sílica. No Parque os táxons foram encontrados em águas com teor de sílica entre 4,9 e $37 \mathrm{mg} . \mathrm{L}^{-1}$, mas com maior incidência em concentrações de sílica maior que 10mg.. $\mathrm{L}^{-1}$, altas concentrações segundo Garrels \& Mackenzie (1971), o que poderia em parte explicar a ocorrência de tantas espécies do gênero registradas presentemente.

Neste estudo foi observada que a morfologia e o tipo de ornamentação da lórica podem variar de acordo com o meio ambiente, fato já apontado por Pringsheim, em 1953, quando trabalhou com culturas.

Em 1992, Menezes realizou amplo estudo da plasticidade morfológica da lórica com oito populações de Strombomonas aplicando métodos numéricos, concluindo que várias espécies deveriam ser englobadas em $S$. verrucosa. Concorda-se com esta autora sob o aspecto do gênero apresentar espécies muito polimórficas e sua sistemática estar alicerçada, fundamentalmente, na morfologia da lórica, acrescendo-se a estes fatos descrições incompletas, o que muitas vezes dificulta sobremaneira as identificações.

O estudo permitiu concluir que existe a necessidade urgente de estudos mais acurados, envolvendo culturas de muitas espécies do gênero, com o objetivo de se trabalhar com populações, o que permitirá verificar polimorfismos, sendo necessário também complementar estes estudos com biologia molecular.

\section{Agradecimentos}

As autoras agradecem a todos os colegas do Museu de Ciências Naturais da Fundação Zoobotânica do Rio Grande do Sul, em especial aos da Seção de Botânica de Criptógamos, pelo auxílio nas coletas; a Fundação de Amparo à Pesquisa do Rio Grande do Sul, pela concessão de bolsa de Iniciação Científica à segunda autora, (proc. 005046/95); à Rejane Rosa, pela cobertura à nanquim dos desenhos.

\section{Referências bibliográficas}

Albuquerque, C.S. \& Menezes, M. 1997. Algas flageladas clorofiladas da área de influência da BR-364, Vilhena Ouro Preto D'Oeste, sudeste do Estado de Rondônia, Brasil. Hoehnea 24(1): 1-16.

Alves-da-Silva, S.M. 1988. Euglenaceae pigmentadas (Euglenophyta) da Estação Ecológica do Tais, Rio Grande do Sul, Brasil. Iheringia, sér. Bot. 38: 109-126.

Alves-da-Silva, S.M. 1998. Levantamento taxonômico e variação temporal das Euglenophyceae de um reservatório raso, no Município de Triunfo, Estado do Rio Grande do Sul. Tese de Doutorado, Instituto de Biociências, Universidade Estadual Paulista. Rio Claro, São Paulo.

Alves-da-Silva, S.M. \& Avila, I.R. 1997. Levantamento florístico das Euglenaceae pigmentadas do Parque Estadual Delta do Jacuí, Rio Grande do Sul, Brasil. Iheringia, sér. Bot. 8: 85-102.

Alves-da-Silva, S.M. \& Crossetti, L.O. 1999. Novas citações de ocorrência de Euglenaceae pigmentadas para o Estado do Rio Grande do Sul, Brasil. Hoehnea 26(1): 47-60.

Alves-da-Silva, S.M. \& Ferraz, G.C. 1991. Euglenaceae pigmentadas de cinco açudes a região carbonífera do município de São Jerônimo, Rio Grande do Sul, Brasil. Hoehnea 18(1): 143-155.

Alves-da-Silva, S.M.; Ferraz, G.C. \& Torres, J.R. 1991. Euglenaceae pigmentadas de dois arroios e Rio Jacuí da região carbonífera do município de São Jerônimo, Rio Grande do Sul, Brasil. Revista Brasileira de Biologia 51(4): 813-828.

Alves-da-Silva, S.M. \& Hahn, A.T. 2004. Study of Euglenophyta in the Jacuí Delta State Park, Rio Grande do Sul, Brazil. 1. Euglena Ehr., Lepocinclis Perty. Acta Botanica Brasilica 18(1): 123-140.

Alves-da-Silva, S.M. \& Torres, J.R. 1994. O gênero Strombomonas no Parque Zoológico, Sapucaia do Sul e no Jardim Botânico, Porto Alegre, Rio Grande do Sul, Brasil. Iheringia, sér. Bot. 44: 15-32.

Balech, E. \& Dastugue, C.E. 1938. Nota preliminar sobre Strombomonas y Trachelomonas. Physis 12: 354-357.

Bicudo, C.E.M.; Ramírez, R.J.J.; Tucci, A. \& Bicudo, D.C. 1999. Dinâmica de populações fitoplanctônicas em ambientes eutrofizado: o Lago das Garças, São Paulo. Pp. 451-507. In: Ecologia de Reservatórios: estrutura, função e aspectos sociais. FAPESP/FUNDIBIO, Botucatu.

Bittencourt-Oliveira, M. 1997. Fitoplâncton do Rio Tibagi, Paraná, Brasil: Nostocophyceae, Chlorophyceae, Euglenophyceae, Crysophyceae e Tribophyceae. Hoehnea 24(1): 1-20.

Bourrelly, P.C. \& Couté, A. 1978. Alges d'eau rares ou nouvelles pour la flora française. Revue Algologique 13(4): 295-307.

Conforti, V.T.D. 1993. Study of the Euglenophyta from Camaleão lake (Amazonas-Brazil). Revue Hydrobiological Tropical 26(3): 187-197. 
Conforti, V.T.D \& Joo, G. 1994. Taxonomic and ultrastructural study of Trachelomonas Ehr. and Strombomonas Defl. (Euglenophyta) from Oxbow lakes in Alabama and Indiana (USA). Cryptogamie, Algologique 15(4): 267-286.

Conforti, V.T.D.; Walne, P.L. \& Dunlap, J.R. 1994. Comparative ultrastructure and elemental composition of envelopes of Trachelomonas and Strombomonas (Euglenophyta). Acta Protozoologica 33: 71-78.

Conrad, W. \& Van-Meel, L. 1952. Materiaux pour une monographie de Trachelomonas Ehrenberg, C., 1934, Strombomonas Deflandre, G., 1930 et Euglena Ehrenberg, C., 1932, genres d'Euglénacées. Mémoires d l'Institute Royal des sciences naturelle Belgique, Sér. 2, 124: 1-176.

Deflandre, G. 1926. Monographie du genre Trachelomonas Ehrenberg. Nemours: André Lesot. 162p., il (cópia xerografica).

Deflandre, G. 1930. Strombomonas, nouveaux genre d'Euglenacées (Trachelomonas Ehr. pro parte). Archiv fur Protistenkunde 69(3): 551-614.

De-Lamonica-Freire, E.M.; Bicudo, C.E.M. \& Castro, A.A. 1992. Ficoflórula do Pantanal de Poconé, estado de Mato Grosso, Brasil, 1: Euglenaceae. Revista Brasileira de Biologia 52(1): 141-149.

Franceschini, I.M. 1992. Algues d'eau douce de Porto Alegre Brésil (les Diatomophycées exclues). Bibliotheca Phycologica 92: 1-81.

Garrels, R. \& Mackenzie, F. 1971. Evolution of sedimentary rocks. W.W. Norton \& Co. Inc, New York.

Huber-Pestalozzi, G. 1955. Euglenaceen. In: G. HuberPestalozzi (ed.). Das Phytoplankton des Susswässers: Systematik und Biologie. Stuttgart: E. Schweizerbart'sche Verlangsbuchhandlug 16(4): 1-605.

Huszar, V.L.M. 1986. Algas planctônicas da lagoa Juturnaíba, Araruama, RJ, Brasil. Rickia 13: 77-86.

Jati, S. \& Train, S. 1994. Euglenaceae pigmentadas de ambientes lênticos da Ilha Porto Rico, município de Porto Rico, Paraná, Brasil. Iheringia, sér. Bot. 45: 117-142.

Keppeler, E.C.; Lopes, M.R.M. \& Lima, C.S. 2002. Ficoflórula do lago Amapá em Rio Branco, Acre, I: Euglenophyceae. Revista Brasileira de Biologia 59(4): 679-686.

Marinho, M.M.; Huzsar, V.L.M. \& Sampaio-Pinto, G.F. 1993. Estrutura da comunidade fitoplanctonica da lagoa de Juturnaíba, Araruama, Rio de Janeiro, Brasil: uma comparação entre os períodos anterior e posterior à construção da barragem do Rio São João. Revista Brasileira de Biologia 53(3): 453-467.

Menezes, M. 1984. Contribuição ao conhecimento das Euglenophyceae pigmentadas (Euglenophyceae) do município do Rio de Janeiro e arredores, Brasil: Estudos taxonômicos. Dissertação de Mestrado, Universidade Federal do Rio de Janeiro, Rio de Janeiro.
Menezes, M. 1992. Taxonomic studies on the Strombomonas verrucosa complex (Euglenophyta). Revista Brasileira de Biologia 52(3): 449-459.

Menezes, M. 1994. Fitoflagelados pigmentados de quatro corpos d'água da região sul do município do Rio de Janeiro, estado de Rio de Janeiro, Brasil. Tese de Doutorado, Instituto de Biociências, Universidade de São Paulo, São Paulo.

Menezes, M. \& Fernandes, V.O. 1987. Euglenaceae (Euglenophyceae) pigmentadas do município de Cáceres e arredores, Mato Grosso, Brasil: uma contribuição a seu conhecimento. Rickia 14: 53-71.

Menezes, M. \& Fernandes, V.O. 1989. Euglenaceae (Euglenophyceae) pigmentadas do noroeste do Estado do Mato Grosso, Brasil: municípios de Barra dos Bugres, Cáceres, Juína e Porto Esperidião. Hoehnea 16: 35-55.

Nevo, E. \& Wasser, S.P. 2000. Biodiversity of cyanoprocaryotes, algae and Fungi of Israel. Cyanoprocaryotes and Algae of Continental Israel. Ruggell: A.R.A. Gantner Verlag.

Pringsheim, G. 1953. Observations on some species of Trachelomonas grown in culture. The New Phytologist 52(2): 93.

Rodrigues, L.C. 1998. Estrutura da comunidade fitoplanctônica de uma lagoa marginal do rio Ivinhema (Lagoa dos Patos, Planície de inundação do alto Rio Paraná) em diferentes períodos do ciclo hidrológico. Dissertação de Mestrado, Universidade Estadual de Maringá, Maringá, PR.

Round, F.E. 1983. Biologia das algas. 2 ed. Ed. Guanabara Dois, Rio de Janeiro.

Souza, C.A. 2002. Taxonomia e aspectos biogeográficos da flora planctônica de um sistema costeiro salobro Lagoa de Carapebus, Município de Carapebus, RJ. Dissertação de Mestrado, Universidade Federal do Rio de Janeiro.

Starmach, K. 1983. Euglenophyta. In: K. Starmach (ed.). Flora Slodkowodna Polski, 3. Polska Academia Nauk, Warszawa.

Tell, G. \& Conforti, V.T.D. 1986. Euglenophyta pigmentadas de la Província del Chaco (Argentina). Nova Hedwigia 41: 353-391.

Tell, G. \& Conforti, V.T.D. 1988. Quelques Strombomonas Defl. (Euglenophyta) de l'Argentina au microscope photonique et életronique à balayage. Nova Hedwigia 46(3-4): 541-556.

Uherkovich, G. \& Raí, H. 1979. Algen aus dem Rio Negro und Nebenflüssen. Amazoniana 4: 611-638.

Uherkovich, G. \& Schmidt, G.W. 1974. Phytoplanktontaxa in dem zentralamazonischen Schwemmlandsee. Amazoniana 2: 243-283.

Xavier, M.B. 1985. Estudo comparativo da flora de Euglenaceae pigmentadas (Euglenophyceae) de lagos do Parque Estadual das Fontes do Ipiranga. Tese de Doutorado. São Paulo, Universidade de São Paulo. 
Xavier, M.B. 1988. O gênero Strombomonas Deflandre de lagos do Parque Estadual das Fontes do Ipiranga, São Paulo, Brasil. Pp.473-505. In: J.G. Tundisi (ed.). Limnologia e Manejo de Represas. EESC/USP/CRHEA/ACIESP, São Paulo.

Xavier, M.B. 1991. Variação sazonal das Euglenaceae pigmentadas de lagos do Parque Estadual das Fontes do Ipiranga, São Paulo, SP. Revista Brasileira de Biologia 51(3): 663-674.
Xavier, M.B. 1993. Distribuição vertical das Euglenaceae pigmentadas do Rio Grande, Represa Billings, São Paulo, Brasil. Acta Limnologica Brasiliensia 6: 11-30.

Zalocar, Y.D. 1991. Strombomonas (Euglenophyta) nuevas e raras de la Argentina. Cryptogamie, Algologie 12(4): 272-281. 